\title{
On-Demand Reversible UV-Triggered Interpenetrating Polymer Network-Based Drug Delivery System Using the Spiropyran-Merocyanine Hydrophobicity Switch
}

Ghani, Mozhdeh; Heiskanen, Arto; Kajtez, Janko; Rezaei, Babak; Larsen, Niels Bent; Thomsen, Peter; Kristensen, Anders; Zukauskas, Airidas; Alm, Martin; Emnéus, Jenny

\section{Published in:}

ACS Applied Materials and Interfaces

Link to article, DOI:

10.1021/acsami.0c19081

Publication date:

2021

Document Version

Peer reviewed version

Link back to DTU Orbit

Citation (APA):

Ghani, M., Heiskanen, A., Kajtez, J., Rezaei, B., Larsen, N. B., Thomsen, P., Kristensen, A., Žukauskas, A., Alm, M., \& Emnéus, J. (2021). On-Demand Reversible UV-Triggered Interpenetrating Polymer Network-Based Drug Delivery System Using the Spiropyran-Merocyanine Hydrophobicity Switch. ACS Applied Materials and Interfaces, 13(3), 3591-3604. https://doi.org/10.1021/acsami.0c19081

\section{General rights}

Copyright and moral rights for the publications made accessible in the public portal are retained by the authors and/or other copyright owners and it is a condition of accessing publications that users recognise and abide by the legal requirements associated with these rights.

- Users may download and print one copy of any publication from the public portal for the purpose of private study or research.

- You may not further distribute the material or use it for any profit-making activity or commercial gain

- You may freely distribute the URL identifying the publication in the public portal 


\title{
On-demand reversible UV-triggered interpenetrating polymer net- work based drug delivery system using the spiropyran-merocyanine hydrophobicity switch
}

\author{
Mozhdeh Ghani ${ }^{1,2}$, Arto Heiskanen ${ }^{2}$, Janko Kajtez ${ }^{2}$, Babak Rezaei ${ }^{3}$, Niels Bent Larsen ${ }^{4}$, Peter Thom- \\ sen $^{1}$, Anders Kristensen ${ }^{4}$, Airidas Žukauskas ${ }^{4}$, Martin Alm ${ }^{1 *}$, Jenny Emnéus ${ }^{2 *}$ \\ ${ }^{1}$ Biomodics ApS, Fjeldhammervej 15, 2610 Rødovre, Denmark \\ ${ }^{2}$ DTU Bioengineering, Building 423, 2800 Kgs. Lyngby, Denmark \\ ${ }^{3}$ DTU Nanolab, Building 345, 2800 Kgs. Lyngby, Denmark \\ ${ }^{4}$ DTU Health Tech, Building 345, 2800 Kgs. Lyngby, Denmark
}

\begin{abstract}
A reversible switchable on-demand UV-triggered drug delivery system (DDS) based on interpenetrating polymer networks (IPNs) with silicone as host polymer and spiropyran (SP) functionalized guest polymer designed and demonstrated. The photo-responsive IPNs provide a new triggered drug delivery concept as it exploits the change in intermolecular interactions (work of adhesion) between drug, matrix and solvent when the incorporated hydrophobic SP moieties transform into the hydrophilic MC form upon light irradiation without degradation and disruption of the DDS. The change of how the copolymer composition (hydrophilicity and content) and the lipophilicity of the drug $(\log \mathrm{P})$ affect the release profile was investigated. A thermodynamic model, based on Hansen solubility parameters (HSP), was developed to design and optimize the polymer composition of the IPNs to get the most efficient light-triggered drug release and suppression of the premature release. The developed IPNs showed excellent result for dopamine, L-dopa and prednisone with around 90-95\% light-triggered release. The model was applied to study the release behavior of drugs with different $\log \mathrm{P}$ and to estimate if the light-induced hydrophobic-to-hydrophilic switch can overcome the work of adhesion between polymer and drug and hence the desorption and release of the drugs. To the best of our knowledge, this is the first time that work of adhesion is used for this aim. Comparing the result obtained from the model and experiment shows that the model is useful for evaluating and estimating the release behavior of specific drugs.
\end{abstract}

\section{INTRODUCTION}

Implantable biomaterials with the capability of providing both an appropriate scaffold for tissue engineering and controlled localized drug delivery have opened new horizons in tissue engineering and regenerative medicine. Such biomaterials are capable of direct cell differentiation through high-performance localized delivery of therapeutic agents and drugs, which have adverse effects or limited effect through systemic drug administration methods, such as oral and intravenous administration ${ }^{1-3}$. Drug delivery systems (DDS) that deliver a therapeutic agent upon a specific stimulus are called triggered DDS. If designed properly a triggered DDS can deliver a drug directly at the point of care at the desired point in time to achieve a localized high concentration of drug while minimizing overall injected dose and systemic concentration. This shows much promise for drugs with high toxicity or narrow therapeutic window. To date, stimuli-responsive DDS have been designed to intelligently switch on/off the release of a drug as the function of responsiveness to the stimuli, which can be endogenous (e.g. $\mathrm{pH}$, enzyme, small bio-molecules, redox and glucose) or exogenous (e.g. magnetic field, temperature, ultrasound, electric pulse and light $)^{4,5}$. Endogenous activation exploits particular physiochemical characteristics of the pathological microenvironment, while exogenous activation provides external stimuli to regulate the release. Each stimulus has both advantages and limitations ${ }^{6}$. Particularly, the control of endogenous stimuli is more difficult because of their tremendous variation from one patient to another, the exogenous stimuliresponsive DDS are more promising for clinical application 7. Among the exogenous stimuli, light is particularly interesting due to its potential to be applied remotely and reversibly with controllable intensity as well as high spatiotemporal precision ${ }^{6,8-10}$. Light-triggered DDS typically consist of a photo-responsive molecular switch as the functional part. This is usually a photochromic chromophore like azobenzene ${ }^{11}$, thymine ${ }^{12}$, o-nitrobenzyl ${ }^{13}$, and spiropyran (SP) ${ }^{14}$. Most of the light-triggered DDS are formulated as degradable photo-responsive films, particles, micelles or liposomes ${ }^{15,16}$. One technique takes advantage of the ability of plasmonic metal nanoparticle clusters to absorb light and convert it into thermal energy, which leads to decomposition of the polymeric film and rupture of the liposomes with subsequent release of the encapsulated drugs ${ }^{15,16}$. Light-triggered micelle-based drug delivery systems are often functionalized with azobenzene or SP which can isomerize upon irradiation with UV light. This causes an alteration in the net dipole moment and a disruption of the micellar hydrophobichydrophilic balance, leading to release of encapsulated contents ${ }^{17,18}$. Among the available classes of light responsive molecular switches, SP ${ }^{19,20}$ has attracted a lot of attention 
21,22, and its photoreaction mechanism thoroughly studied, and exploited ${ }^{22,23}$ for construction of different hybrid photoswitchable structures ${ }^{21,22,24-26}$. SP is unique in that its two isomers exhibit dramatically different physicochemical properties in response to light and that the isomerisation is reversible ${ }^{21,22}$. Under visible (Vis) light or at dark, the molecule is in its SP form, which is colourless, hydrophobic, nonpolar, uncharged, and insoluble in water. Under UV light irradiation, it isomerizes to the merocyanine (MC) form, which is coloured (violet), hydrophilic, polar, zwitterionic and soluble in water ${ }^{27,28}$ (Figure 1a). Several scientists have been inspired by the hydrophobicity switch between the SP and $\mathrm{MC}$ isomers and its quick transition rate ${ }^{29}$, which has led to various potential applications, such as bioimaging ${ }^{30}$, sensors ${ }^{28,31,32}$, controlled release ${ }^{14,18,33}$, optical switching displays ${ }^{34}$, and optical data storage ${ }^{35,36}$. The use of SP for light-triggered drug delivery has gained particular attention during recent decades ${ }^{14,17,18,29,37-41}$. This research area is still in its early stages, as no commercial product has emerged, but the amount of conducted research indicates the huge potential to use SP for light-triggered DDSs ${ }^{28}$. This research has mainly focused on the formation of SP-containing amphiphilic molecules that self-assemble into micelles or liposomes. Upon UV exposure the molecules loses their amphiphilic nature and the micelles/liposomes are disrupted and the drug cargo is released ${ }^{18}$. Another common approach is to embed the drug in mesoporous silica nanoparticles which are modified with SP as a gate molecule on their surface, which upon UV exposure transforms the hydrophobic SP into the hydrophilic MC form, resulting in the diffusion and release of the embedded drug ${ }^{39,42-44}$.

Most of the light-triggered DDS described in literature are based on disassembly and disruption of the drug carriers, which suffer from premature drug leakage (premature release) before reaching the target site. Furthermore, these systems lack the reversibility, i.e. they do not possess the capability to stop the release again after the initial trigger. Also, the residual carrier segments, formed through the disruption process, are not desired in terms of toxicity. Therefore, it would be convenient to develop a reversible light-triggered DDS that exploits the change in the intermolecular interactions (work of adhesion) between polymer, drug and solvent, without the disruption of the system. Moreover, a wellknown issue with light-triggered DDS (like the SP-MC system) is that most respond to UV light, which can be harmful to tissues, and has a low penetration depth (around $10 \mathrm{~mm}$ ) ${ }^{6,45,46}$. Effort has therefore been invested to address these limitations by replacing UV with two-photon NIR light in the range of 650-900 nm ${ }^{4,6,47}$. Alternatively, a light source can be implanted locally inside the body, as recently proposed ${ }^{48}$. We have previously employed supercritical carbon dioxide $\left(\mathrm{scCO}_{2}\right)$ technology to generate IPNs by impregnating silicone elastomers (the host polymer) with different guest polymers formed by free radical polymerization ${ }^{49-55}$. This technology is capable of incorporating different types of monomers into the silicone to develop IPNs with diverse properties and good potential for long-term continuous drug delivery ${ }^{53-55}$ and their performance at in vivo physiological conditions has been demonstared in a previous study ${ }^{50}$. However, to the best of our knowledge, there is no systematic study of the $\mathrm{scCO}_{2}$ technology to develop a photo-switchable silicone-based IPN as a light-triggered DDS.
Here we describe the development of a light-triggered DDS based on IPNs that include copolymers of the photochromic compound spiropyran methacrylate (SPMA) as the guest polymer and evaluation of the triggered-release of five different drugs with varying hydrophobicity (log P value). Silicone elastomer was impregnated with different photochromic guest polymers including SP co-polymerized with different acrylic monomers with varying hydrophilicity; 2hydroxyethyl methacrylate (HEMA), poly (ethylene glycol) methyl ether acrylate (PEGMEA) and butyl methacrylate (BMA).

A thermodynamic model based on HSP was developed to estimate the work of adhesion between each drug and guest polymer and used to design, tailor and optimize the guest polymer composition. The mechanism of light-triggered drug release is a hydrophobic-to-hydrophilic switch of the IPNs where the release of the drug depends on the intermolecular interactions between the drug, the guest polymer and the solvent. Therefore, by applying the model and altering the composition of the guest copolymers with varying hydrophilicity, the surface tension was tailored to ensure a triggered release and suppress premature release. The IPNs were characterized using FTIR and XPS to confirm the presence of guest polymer in the surface and bulk of the material, as well as by water uptake measurements. Fluorescence spectroscopy was applied to demonstrate the photochromic properties of the IPNs. Furthermore, the thermodynamic model was applied to estimate the work of adhesion between each drug and guest polymer and to predict the release behaviour of the drugs from the developed photoactive IPNs. To the best of our knowledge this is the first time that work of adhesion is used for this aim.

Finally, we performed some initial cell viability and biocompatibility testing of the IPNs before and after UV treatment by culturing human neural stem cells (hNSCs) (live/dead cell staining) and their further 10 days differentiation on the IPNs (immunocytochemistry).

\section{EXPERIMENTAL SECTION}

2.1. Materials and chemicals. Silicone hollow cylinders (L:22 mm, ID: $4 \mathrm{~mm}$, WT: $0.5 \mathrm{~g}$ ) were cut from extruded silicone tubing (Nusil MED-4720) and carbon dioxide (N48) was supplied by Air Liquide Denmark (Copenhagen, Denmark). 2-hydroxyethyl methacrylate (97\%, HEMA), poly (ethylene glycol) methyl ether acrylate Mn 480 (PEGMEA), butyl methacrylate (BMA), 2,3,3-Trimethylindolenin 98\%, 2-hydroxy-5-nitrobenzaldehyde, acetonitrile, diethyl ether, dichloromethane (DCM), hexane, potassium hydroxide, triethylamine, methacryloyl chloride, 2-bromoethanol, 98\% ethylene glycol dimethacrylate (EGDMA), tetrahydrofuran (THF), doxycycline hyclate, curcumin, dopamine hydrochloride and ethanol $(99.8 \%, \mathrm{EtOH})$ and 3-(3,4-Dihydroxyphenyl)-L-alanine (L-DOPA) were purchased from SigmaAldrich. Removal of the monomethyl ether hydroquinone (MEHQ) inhibitor from HEMA and PEGMEA and their purification was done as described previously ${ }^{53}$. Diethyl peroxydicarbonate (DEPDC) in hexane was prepared as described by $\mathrm{Xu}$ et al ${ }^{56}$ and used as initiator for radical polymerization.

2.2. Synthesis of spiropyran methacrylate (SPMA) monomer (1'-(2-methacryloxyethyl)-3',3'-dimethyl-6-nitrospiro-(2H-1 benzopyran-2,2'-indoline)). In order to fabri- 
cate the photo-responsive IPNs, the photochromic compound, 2-(3',3'-Dimethyl-6-nitro-3'H-spiro[chromene-2,2'indol]-1'-yl)-ethanol (SPOH) was synthesized according to a previously published procedure ${ }^{57}$. The SPMA monomer was synthesized through modification of SPOH with methacryloyl chloride according to the method described in ${ }^{58}$. The HNMR spectra of samples were obtained using a $400 \mathrm{MHz}$ Bruker Avance III NMR spectrometer with $\mathrm{CD}_{3} \mathrm{CN}$ and $\mathrm{CDCl}_{3}$ as the solvent of SPOH and SPMA, respectively. The spectra were processed using TOPSPIN 4.0.2 software (Bruker) and are shown in Figure S1 and S2 in Supporting information (SI). The H-NMR spectra of the synthesized compounds showed the expected peaks and chemical shifts according to the reference ${ }^{59}$, which confirms the structure and purity of them. The photochromic properties of the synthesized SP is presented in the S10 of the SI.

2.3. Fabrication of photo-responsive IPNs. IPNs containing photochromic acrylic-based guest polymers were prepared in $\mathrm{scCO}_{2}$ by impregnating the silicone elastomer with the guest monomers ${ }^{49-55}$, including the photochromic monomer SPMA, in 16 mL-custom-made stainless-steel highpressure reactors (Abeto, Copenhagen, Denmark). Hydrophilic and hydrophobic IPNs were fabricated by co-polymerization of HEMA, PEGMA, and SPMA into poly(HEMAco-PEGMEA-co-SPMA) and copolymerizing BMA and SPMA into poly(BMA-co-SPMA) as the guest polymer, respectively. The combination of BMA, HEMA and SPMA was used to fabricate three partially hydrophilic poly(BMAco-HEMA-co-SPMA) IPNs with different HEMA molar ratio of $8.5,12$ and $24 \%$. Briefly, a mixture of monomers solution (the composition of the monomers solution for each IPN is described in S2 (SI)), initiator $(0.8 \mathrm{~mL}$ of $0.2 \mathrm{M}$ DEPDC) and crosslinker (0.096 mL of EDGMA) with approximately $0.5 \mathrm{~g}$ silicone hollow cylinders were added to the reactors. Then the reactors were closed and pressurized with $\mathrm{CO}_{2}$ to 400 bar at $40{ }^{\circ} \mathrm{C}$. After $16 \mathrm{~h}$ the pressure is slowly released (approx. $30 \mathrm{~min}$ ) and the reactor is allowed to return to ambient temperature. The samples are purified by extracting the residual monomers and un-crosslinked polymers by soaking in $96 \%$ ethanol for 7 days and subsequent drying at $40{ }^{\circ} \mathrm{C}$ for 5 hours. The guest polymer content $(G)$ of the IPNs were calculated using eq. (1).

$$
G(\%)=\left(\frac{m_{I P N}-m_{\text {Silicone }}}{m_{I P N}}\right) \times 100
$$

Where, $m_{I P N}$ is the mass of the IPN samples and $m_{\text {silicone }}$ is the mass of pristine silicone before impregnation with the guest polymer.

2.4. IPN characterization. The photochromic properties of the IPNs were evaluated by recording fluorescence spectra using an Andors Shamrock SR303i spectrometer equipped with UV LED lamp (375 $\mathrm{nm}$ ) and a laser excitation source (Cobolt $532 \mathrm{~nm}$ ). For the isomerization reaction (SP to MC), the excitation was performed by a UV LED lamp using 0.52 $\mathrm{mW}$ output and $400 \mathrm{~mA}$ current. For the reverse reaction (MC to SP), fluorescence spectra were acquired at an excitation wavelength of $532 \mathrm{~nm}$. The exposure time for UV and Vis light was set to $6 \mathrm{~min}$ for the experiments. To investigate the reversibility of photo-isomerization in response to alternating light irradiation, eight cycles of sequential UV and Vis light exposure were applied to the IPN sample, and the fluorescent emission was recorded accordingly. The atomic composition of the surface and bulk of pristine silicone and IPNs with and without SP was analysed by X-ray photoelectron spectroscopy (XPS), using a K-Alpha spectrometer (Thermo Fisher Scientific Inc., USA) equipped with a monochromatic Al Ka X-ray source (pass energy: $200 \mathrm{eV}$ for survey spectra). The atomic percentages of elements were extracted using the software package (Avantage Thermo VG), provided by ThermoFisher Scientific. Attenuated total reflection Fourier transform infrared spectroscopy (ATRFTIR, PerkinElmer Technologies, USA) was used to identify different functional groups and verify the presence of guest polymers in the IPNs.

The light-triggered release from photoactive IPNs with varying hydrophilicity of the guest polymer were investigated by examining the release behaviour of five drugs with varying lipophilicity (different $\log \mathrm{P}$ values) with and without UV irradiation.

2.5. Drug loading and drug release. The hollow cylinder IPNs $(1.00 \mathrm{~cm})$ were loaded by soaking them in $5 \mathrm{~mL}$ of 12.5 $\mathrm{mg} / \mathrm{mL}$ drug solution in $70 \%$ ethanol for 1 week at $4^{\circ} \mathrm{C}$. It was previous shown that the IPNs were fully loaded after 34 days ${ }^{50}$. To remove loosely attached drug from the IPN samples, they were vortex-washed five times $(10 \mathrm{sec}$ each time) with fresh miliQ water. For characterization of drug release and the effect of UV irradiation on the release behaviour, IPNs were immersed in vials containing $5 \mathrm{~mL}$ of PBS (0.1 M, pH 7.2). The UV irradiation of the samples was done using a UV lamp (6-watt, $375 \mathrm{~nm}$, Analytik Jena, Germany). $300 \mu \mathrm{L}$ of the release medium was sampled from each vial at different time intervals. UV-Vis absorbance of the solution was then immediately recorded at the maximum absorption wavelength. Table 1 represents the properties of the drugs tested in this work.

Table 1. The properties of the tested drugs

\begin{tabular}{|c|c|c|c|c|c|c|}
\hline \multirow{3}{*}{ Drug } & \multirow{3}{*}{$\begin{array}{c}\text { Log } \\
\mathrm{P}\end{array}$} & \multirow{3}{*}{$\begin{array}{c}\text { Water } \\
\text { solubility } \\
\mathrm{mg} / \mathrm{mL}\end{array}$} & \multirow{3}{*}{$\begin{array}{c}\lambda_{\max } \\
\mathrm{nm}\end{array}$} & \multicolumn{3}{|c|}{ HSP } \\
\hline & & & & $\delta_{\mathrm{d}}$ & $\delta_{\mathrm{p}}$ & $\delta_{\mathrm{h}}$ \\
\hline & & & & \multicolumn{3}{|c|}{$\mathrm{MPa}^{1 / 2}$} \\
\hline $\begin{array}{l}\text { Doxycy- } \\
\text { cline hyclate }\end{array}$ & -1.9 & 50 & 344 & 24.7 & 8.49 & 22.2 \\
\hline Dopamine & $\overline{-}-$ & $\begin{array}{l}\text { Freely } \\
\text { soluble }\end{array}$ & 284 & 18.2 & 10.3 & 19.5 \\
\hline $\begin{array}{c}\text { Levodopa } \\
\text { (L-dopa) }\end{array}$ & 0.05 & 5 & 284 & 22.34 & 6.26 & 21.86 \\
\hline Prednisone & 1.46 & $0.22-0.24$ & 282 & 21.52 & 8.88 & 16.26 \\
\hline Curcumin & 3.62 & 0.0057 & 426 & 18.8 & 7.7 & 11.1 \\
\hline
\end{tabular}

2.6. Cell viability and immunocytochemistry. The in vitro biocompatibility and the ability of photoactive IPNs to be used asa substrate for stem cell differentiation was assayed through differentiating hNSCs of ventral mesencephalic origin on the poly(BMA-co-HEMA-co-SPMA) IPN with 8.5\% HEMA and poly(BMA-co-SPMA) IPN. Following purification and sterilizing with ethanol, the IPNs were rinsed with sterile MQ water, incubated in a cell culture medium overnight then coated with Geltrex (ThermoFisher Scientific). hNSCs cells were cultured on Geltrex coated IPNs, as previously described ${ }^{60,61}$. Cells were seeded in growth medium containing DMEM/F12 with GlutaMAX, $0.5 \%$ w/v 
AlbuMAX, 5 mм HEPES (all from ThermoFisher Scientific), $6 \mathrm{~g} / \mathrm{L}$ glucose (Sigma Aldrich), $40 \mu \mathrm{M}$ each of each Lasparagine monohydrate (MerckMillipore), L-alanine (MerckMillipore), L-glutamin, L-aspartic acid (MerckMillipore), L-proline (MerckMillipore), acid (MerckMillipore) and 100x diluted N-2 supplement (ThermoFisher Scientifica), penicillin/streptomycin mix, $20 \mathrm{ng} / \mathrm{L}$ each of fibroblasts growth factor (FGF) and epidermal growth factor (EGF) (R\&D systems). Viability of the hNSC cells was assessed 24 hours after seeding using a live/dead cell viability kit (calcein AM and ethidium homodimer-1 (ThermoFisher Scientific)), according to the manufacturer's instructions. Cells were incubated with ethidium homodimer-1 and calcein AM for half an hour at $5 \% \mathrm{CO}_{2}$ and $37{ }^{\circ} \mathrm{C}$ to stain dead (red) and live cells (green), respectively. Then IPN samples were washed with PBS and imaged using an LSM 710 (Carl Zeiss) inverted confocal laser scanning microscope. For stem cell differentiation and immunocytochemistry, growth medium was replaced with differentiation medium 24 hours after seeding. Differentiation medium components were the same as growth media, except that EGF and FGF were substituted by $1 \mathrm{mM}$ Dibutyryladenosine 3',5'-cylic monophosphate sodium salt (Sigma Aldrich) and $2 \mathrm{ng} / \mathrm{L}$ GDNF (PeproTech). After 48 hours, the differentiation medium was entirely exchanged with fresh differentiation medium. Then every second day, 2/3 differentiation medium was exchanged with fresh differentiation medium. At the experimental endpoint (differentiation day 10), 4\% paraformaldehyde was used for fixation of cells. Before immunocytochemistry staining, the samples were incubated with blocking solution (1\% goat serum and $1 \%$ horse serum in $0.3 \%$ Triton X-100 in PBS) to permeabilize the cell membranes and prevent unspecific binding of antibodies. Then the samples were stained with primary antibodies by incubating them in anti- $\beta$-tubulin (mouse, Sigma-Aldrich T8660, 1:500) and anti-tyrosine hydroxylase (rabbit, PelFreez Biologicals P40101, 1:500) in blocking solution overnight at 4 ${ }^{\circ} \mathrm{C}$. After rinsing of samples with PBS, they were stained with secondary antibodies by incubating in Alexa 546 goat anti-mouse (ThermoFisher A-11030, 1:500) and Alexa 647 goat anti-rabbit (ThermoFisher A-21245, 1:500) in blocking solution for 2 hours at room temperature. The cell nuclei were counterstained with $2 \mu \mathrm{g} / \mathrm{ml}$ Hoechst 33342 (ThermoFisher H1399) for 15 min followed by rinsing and incubation in PBS. LSM 710 (Carl Zeiss) inverted confocal laser scanning microscope was used for acquiring the images using objectives with $40 \mathrm{x}$ magnification. Z-stacks were acquired in order to visualize the cells growing at different focal planes. Images were processed and analyzed using ImageJ software.

\section{RESULTS AND DISCUSSION}

3.1 Development of the thermodynamic model. Figure $1 \mathrm{~b}$ illustrates the release process from an IPN. It shows, from a thermodynamic point of view, what happens upon UV irradiation at the interphase between drug, guest polymer and solvent. The desorption and triggered release of a drug from the IPN can be described in terms of the involved surface energy changes. The work of adhesion $\left(W_{P D}\right)$ between a guest polymer $(P)$ and drug $(D)$ can be estimated according to eq. (2) ${ }^{62}$ by considering the relationship between the interfacial surface tension between the drug, polymer and solvent (water, $W$ ).
$W_{P D}=\gamma_{D W}+\gamma_{P W}-\gamma_{P D}$

Where, $\gamma$ is the interfacial surface tension between two substances, and P, D and $\mathrm{W}$ denote the polymer, drug and solvent (water), respectively.

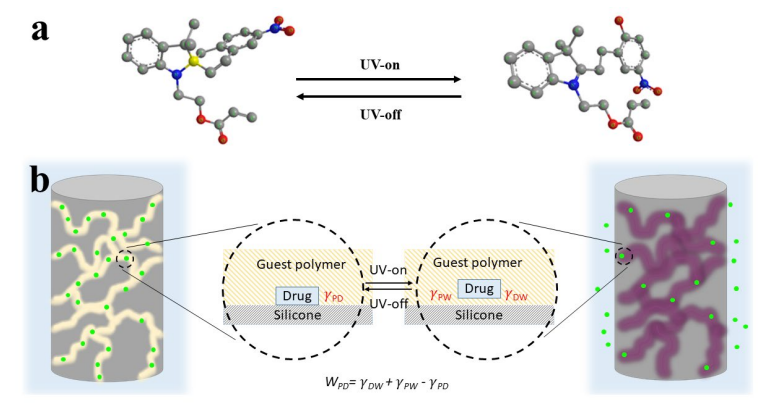

Figure 1. a) Photochromism of spiropyran in the guest polymer network and reversible transformations between the the hydrophobic SP and the hydrophilic MC, b) Desorption and release of drug molecules from photoactive guest polymer in an IPN.

The work of adhesion is the minimum work required to separate two adjacent phases in contact and the energy needed to break the adhesive bonds ${ }^{63,64}$. Therefore, the work of adhesion is an interesting thermodynamic parameter that can be used to predict if a certain drug will adsorb to a certain polymer in a certain environment. Decreasing the work of adhesion between a polymer and a drug means that it becomes more energetically favourable for the drug to desorb from the polymer surface. Adsorption and desorption of a drug to or from the surface of a material (e.g. a guest polymer in an IPN) can be described by the intermolecular interactions between the drug, polymer and solvent. The thermodynamic model based on the interrelation of HSP ${ }^{65}$ and the work of adhesion ${ }^{64}$ was developed to estimate the intermolecular interactions between drug, polymer and solvent. Thus, the model could be used to tailor and optimize the polymer composition of the drug carrier (e.g. IPN) and also to predict whether the release of specific drugs with different $\log \mathrm{P}$ values can be triggered by light, i.e. if the change in work of adhesion caused by irradiation of light is enough to trigger the desorption and release of a drug from the SPbased IPN.

The HSP gives a systematic estimate of the compatibility between two compounds. It describes the cohesive energy that holds molecules together to form materials or liquids, and is equal to the energy needed for vaporization (breaking all the cohesive bonds). This energy arises from dispersion forces $(d)$, polar forces $(p)$ and hydrogen bond forces $(h){ }^{65}$ with the total cohesive energy $E(\mathrm{~J} / \mathrm{mol})$, which is the sum of these individual energies, as given by eq. (2) ${ }^{65}$.

$$
E=E_{d}+E_{p}+E_{h}
$$

The HSP $(\delta)$ (with the unit $\mathrm{MPa}^{1 / 2}$ ), can be obtained through eq. (4), where $V$ is the molar volume $(\mathrm{mL} / \mathrm{mol})$, and the square of $\delta$ is the sum of the square of the individual energy $\delta_{\mathrm{d}}, \delta_{\mathrm{p}}$, and $\delta_{\mathrm{h}}$, arising from dispersion-, polar- and hydrogen bond forces, respectively ${ }^{65}$, as shown in eq. (5).

$$
\delta^{2}=E / V
$$




$$
\delta^{2}=\delta_{d}^{2}+\delta_{p}^{2}+\delta_{h}^{2}
$$

The surface tension ( $\gamma$ in eq (2)) of matter originates from the cohesive forces holding this matter together ${ }^{65}$. Consequently, the surface tension of a pure substance $\left(\gamma_{1}\right)$ can be estimated by applying HSP where the correlation between $\gamma_{1}$ and HSP is given by eq. (6) ${ }^{66}$.

$$
\gamma_{1}=k_{1} \mathrm{~V}^{\frac{1}{3}}\left[\left(\frac{\delta_{d}}{k_{3}}\right)^{2}+k_{2}\left(\left(\frac{\delta_{p}}{k_{3}}\right)^{2}+\left(\frac{\delta_{h}}{k_{3}}\right)^{2}\right)\right]
$$

Using the correlation between $\gamma$ and HSP, considering the dispersion forces $(d)$, polar forces $(p)$ and hydrogen bonding $(h)$ as interfacial interactions and cohesive forces, eq. (7) can be derived by calculating the work of adhesion based on the HSP. The full derivation is described in detail in S3 of the SI.

$$
\begin{aligned}
& W_{P D}=2\left(\gamma_{W}-\frac{k_{1}}{k_{3}{ }^{2}} V_{D}^{\frac{1}{6}} V_{W}^{\frac{1}{6}} \delta_{d D} \delta_{d W}-\right. \\
& \frac{k_{1}}{k_{3}{ }^{2}} V_{P}^{\frac{1}{6}} V_{W}^{\frac{1}{6}} \delta_{d P} \delta_{d W}+\frac{k_{1}}{k_{3}{ }^{2}} V_{P}^{\frac{1}{6}} V_{D}^{\frac{1}{6}} \delta_{d P} \delta_{d D}- \\
& \frac{k_{1} k_{2}}{k_{3}{ }^{2}} V_{D}^{\frac{1}{6}} V_{W}^{\frac{1}{6}} \delta_{P D} \delta_{P W}-\frac{k_{1} k_{2}}{k_{3}{ }^{2}} V_{P}^{\frac{1}{6}} V_{W}^{\frac{1}{6}} \delta_{P P} \delta_{P W}+ \\
& \frac{k_{1} k_{2}}{k_{3}{ }^{2}} V_{P}^{\frac{1}{6}} V_{D}^{\frac{1}{6}} \delta_{P P} \delta_{P D}-\frac{k_{1} k_{2}}{k_{3}{ }^{2}} V_{D}^{\frac{1}{6}} V_{W}^{\frac{1}{6}} \delta_{h D} \delta_{h W}- \\
& \left.\frac{k_{1} k_{2}}{k_{3}{ }^{2}} V_{P}^{\frac{1}{6}} V_{W}^{\frac{1}{6}} \delta_{h P} \delta_{h W}+\frac{k_{1} k_{2}}{k_{3}{ }^{2}} V_{P}^{\frac{1}{6}} V_{D}^{\frac{1}{6}} \delta_{h P} \delta_{h D}\right)
\end{aligned}
$$

where $V$ is the molar volume of a substance $(\mathrm{mL} / \mathrm{mol}), \delta$ is the HSP of a specific type of molecular interaction $(\sqrt{\mathrm{MPa}})$, and $k_{1}$ is Beerbower's constant which has a value of 0.0715 67. $k_{3}$ is a conversion constant with the value of $2.0455{ }^{65}$. To determine the value of constant $k_{2}$, the theoretical surface tension of 132 solvents with known HSP ${ }^{65}$ and surface tensions ${ }^{68}$ at $25^{\circ} \mathrm{C}$ were calculated using eq. (6). Based on this, the root mean square error (RMSE) between the calculated and known surface tensions was determined and minimized by changing the value of $k_{2}$ using Excel's solver function. This yields a value for $k_{2}$ of 0.5793 with an RMSE of 6.6. By applying eq. (7) using known and calculated values, it is possible to determine the work of adhesion $\left(W_{P D}\right.$ in $\mathrm{mN} /$ $\mathrm{m}$ ) between a polymeric matrix and a drug in a given solvent. By evaluating the work of adhesion of different drugs with different polymers in different solvents it is possible to intelligently choose a suitable combination to meet the specification of the DDS. This is especially important when designing a triggered DDS.

The validity of this new thermodynamic model was demonstrated when applied for designing the guest polymer composition of SP-based IPN and further evaluated with five different drugs.

3.2. Designing the guest polymer composition of IPNs using the thermodynamic model. The developed thermodynamic model (eq. (7)) was applied to design and optimize the guest polymer composition to minimize the premature release. For this study, doxycycline (DOX) was used as a model drug. The HSP of different hydrophilic and hydrophobic monomers (BMA, HEMA, SPMA, PEGMEA) was estimated using the group contribution method ${ }^{69,70}$ using van krevelen's ${ }^{70}$ values (Table S1 in SI) and presented in Table S2 (SI). The HSP of copolymers, used as guest polymers of the IPNs, was calculated using the molar fraction of monomers $\left(x_{i}\right)$ and their partial solubility parameters $\left(\delta_{i}\right)$ in Table S2 (SI), according to eq. (8) ${ }^{71}$, and presented in Table S3 (SI).

$$
\delta_{\text {copolymer }}=\sum_{i} x_{i} \delta_{i}
$$

The work of adhesion between DOX and different guest polymer compositions of varying hydrophilicity was determined by introducing the HSP values in Table S3 (SI) into eq. (7) and presented in Table 2.

Table 2. Estimated work of adhesion $(\mathrm{mN} / \mathrm{m})$ between DOX and different guest polymer composition.

\begin{tabular}{lc}
\hline \multicolumn{1}{c}{ Guest polymer composition and DOX } & $\begin{array}{c}\text { Work of } \\
\text { adhesion }\end{array}$ \\
\hline Poly(BMA-co-SPMA) & 31.22 \\
Poly(HEMA-co-PEGMEA-co-SPMA) & 15.75 \\
Poly(BMA-co-HEMA-co-SPMA), (8.5\% & 29.04 \\
HEMA) & \\
Poly(BMA-co-HEMA-co-SPMA), (12\% & 28.54 \\
HEMA) & \\
Poly(BMA-co-HEMA-co-SPMA), $(24 \%$ & 25.86 \\
HEMA) & \\
\hline
\end{tabular}

From the thermodynamic point of view at the interphase between of the drug, guest polymer and water (Figure 1), a high work of adhesion between the drug and guest polymer indicates that it is more energetically favourable for the drug to adsorb to the guest polymer, which should lead to less premature release when UV is off, and vice versa, a low work of adhesion should lead to larger premature release. Therefore, from the model-estimated work of adhesion between DOX and different guest polymers in the Table 2, it is inferred that the poly(HEMA-co-PEGMEA-co-SPMA) IPN and poly(BMA-co-SPMA) IPN should have the highest and lowest premature release, respectively. Moreover, by increasing the HEMA ratio in the guest polymer composition the premature release should increase. To validate the performance of the model in predicting the release behaviour, the triggered release of DOX from all five IPNs with different guest polymer composition was studied (section 3.5).

3.3. Fabrication and characterization of the photo-responsive IPN. The guest polymer content of the produced poly(HEMA-co-PEGMEA-co-SPMA) and poly(BMA-coSPMA) IPNs was determined to be $45 \%$ and $28 \%(\mathrm{w} / \mathrm{w})$, respectively. The guest polymer content of poly(BMA-coHEMA-co-SPMA) IPNs with HEMA molar ratio of $8.5 \%$, $12 \%$ and $24 \%$ was determined to be $35 \%, 37 \%$ and $49 \%$ $(\mathrm{w} / \mathrm{w})$, respectively. Figure 2 shows photos of the synthesized IPNs with different guest polymers. As can be seen, already after $20 \mathrm{sec}$ UV irradiation of the three types of IPNs, a deep colouration of the samples can be observed, indicating that the isomerization of SP to MC is working in the IPNs.

Figure 3A compares the ATR-FTIR spectra of pristine silicone, SPOH and poly (BMA-co-HEMA-co-SPMA) IPN, HEMA 8.5\%. The results for other IPNs are shown in Figure 
4S (SI). The characteristic peaks of silicone elastomer assigned to $\mathrm{Si}-\mathrm{O}-\mathrm{Si},-\mathrm{CH} 3$ and $\mathrm{Si}-\mathrm{CH} 3$ groups (labelled on the

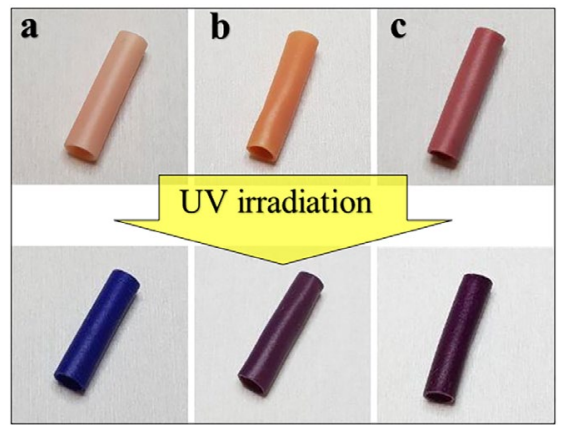

Figure 2. $20 \mathrm{sec}$ UV irradiation changes the color of photo-responsive IPNs with guest polymer of: a) poly(BMA-co-SPMA), b) poly(BMA-co-HEMA-co-SPMA) with a BMA/HEMA ratio of 11/1, and c) poly(HEMA-co-PEGMEA-co-SPMA), due to isomerization of SP to MC.

spectra) are observed for all the IPNs, owing to the presence of silicone in their structure. A characteristic peak at around $1720 \mathrm{~cm}^{-1}$ can be identified for all IPNs, which corresponds to the ester carbonyl stretching vibration of acrylate group in the guest polymers. IPNs containing HEMA has an additional broad peak around $3300 \mathrm{~cm}^{-1}$ due to the terminal hydroxyl groups. FTIR spectrum of SPOH exhibits a band at $1651 \mathrm{~cm}^{-1}$ and $1576 \mathrm{~cm}^{-1}$ which is assigned to the stretching vibration of endocyclic $\mathrm{C}=\mathrm{C}$ and aromatic carbon, respectively ${ }^{72}$. The same peaks appeared in the spectra of all IPNs which clearly indicates the presence of SPMA in their structure. Preliminary tests was also performed with two-photon NIR $(750 \mathrm{~nm})$ to see if the isomerization of SP to MC could be achieved this way. Figure S8 in (S9 in SI) indicates that the IPNs could in principle be excited with two-photon NIR and potentially used to trigger the release of drugs.

The XPS spectra of pristine silicone, IPN with poly(BMAco-HEMA-co-SPMA) as guest polymer and the same IPN but without SPMA as the control sample are shown in Figure 3B. The XPS spectra of the surface (Figure $3 \mathrm{Ba}$ ) shows a nitrogen $\left(\mathrm{N} 1_{\mathrm{s}}\right)$ peak at $398 \mathrm{eV}$ for the photoactive IPN containing SMPA, whereas for pristine silicone and the IPN

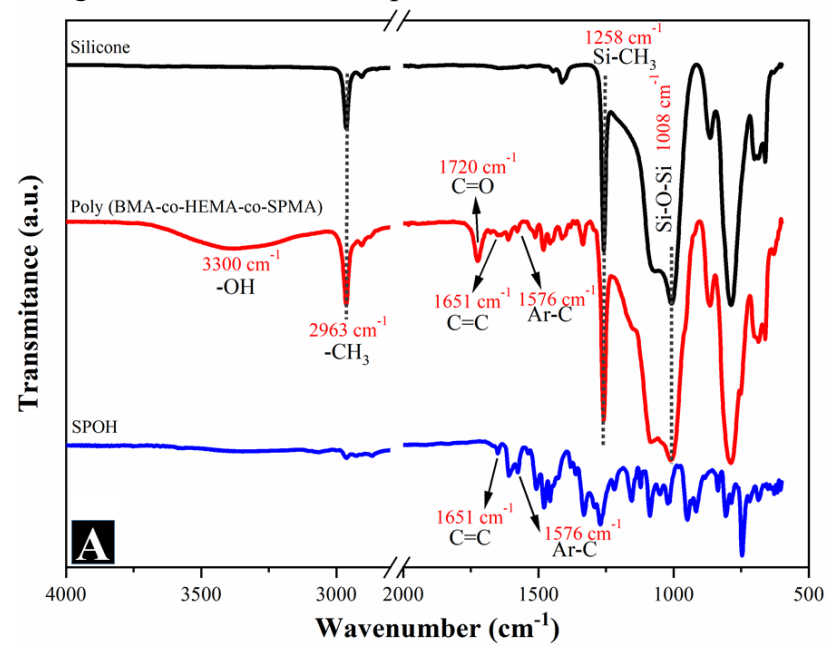

without SPMA, no nitrogen element was detected. The existence of nitrogen on the surface of the IPN could be ascribed to the presence of the photochromic SP moiety on the surface. Figure $3 \mathrm{Bb}$ shows the XPS sputter depth profile of the nitrogen element for the photoactive IPN sample. The inset of Figure $3 \mathrm{Bb}$ shows the intensity of nitrogen peak as the function of etching sequence; the approximately constant intensity of the nitrogen element within the interior structure of the IPN confirms the uniform distribution of SPMA throughout the IPN. Moreover, when an IPN was cross-cut and exposed to UV light, the colour immediately changed to purple throughout the cross-section (Figure $3 \mathrm{Bc}$ ). This confirms the presence of SPMA inside the IPN, which is consistent with the XPS observations. To investigate the photochromic properties of the IPNs, they were subsequently exposed to UV and Vis light. The UV light prompted a fast uniform deep colouration of the IPNs (inset of Figure 4a), which is due to the structural conversion from a colourless hydrophobic SP form to a purple-coloured hydrophilic MC form through the cleavage of the spiro carbon-oxygen bond. This observation shows that the photoactivity of SP was not destroyed during the production process. The reversibility of the photo-isomerization of the IPNs (i.e. MC to SP) was demonstrated by the colour-shift back to the initial colour when irradiated by Vis light for 6 min (inset of Figure $4 b)$. The fact that the SP form is non-fluorescent, whereas the $\mathrm{MC}$ form emits fluorescence ${ }^{73}$ can be used to study the reversible isomerization of SP moiety to MC inside the IPNs by fluorescence spectrophotometry. The fluorescence spectra of poly(BMA-co-HEMA-co-SPMA) IPN is shown in Figure $4 \mathrm{a}$ and $\mathrm{b}$ for the isomerization reaction (SP to $\mathrm{MC}$ ) induced by UV irradiation ( $375 \mathrm{~nm}$; Figure $4 \mathrm{a}$ ) and the reverse reaction (MC to SP) by Vis light (532 nm; Figure 3b) irradiation. Figure $4 \mathrm{a}$ shows that there is no fluorescence before exposure to UV light, whereas after irradiation with UV light, emission at $651 \mathrm{~nm}$ immediately appears, indicating that the SP form was converted to the MC form. It can be seen that the fluorescence intensity increases with irradiation time (spectra recorded every $20 \mathrm{~s}$ ), almost reaching a plateau after $6 \mathrm{~min}$. This indicates that the IPN reached a photo-stationary state and the inset of figure $4 \mathrm{a}$, also shows the IPN colour turns into purple.

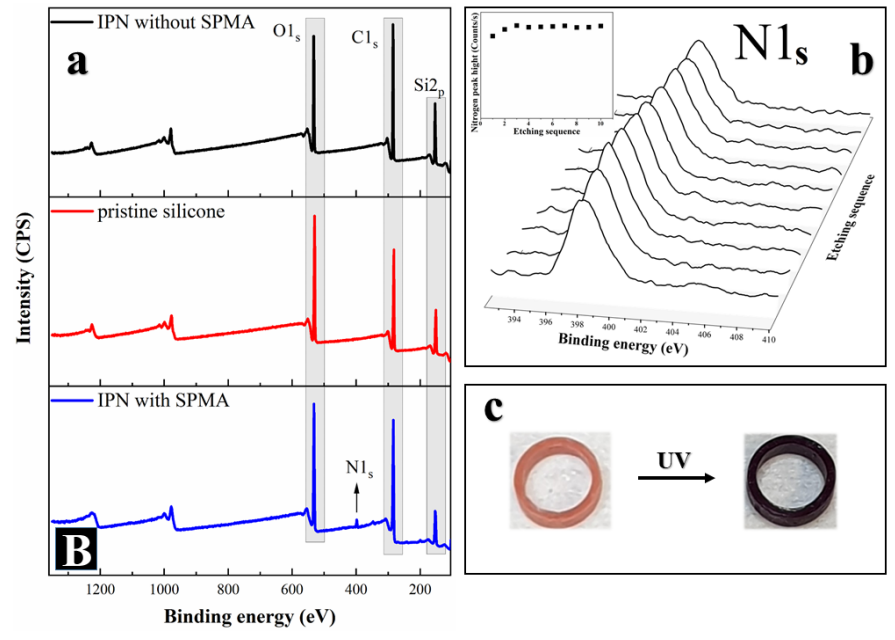

Figure 3. A) Transmission FTIR spectra of pristine silicone, poly (BMA-co-HEMA-co-SPMA) IPN and SPOH. Ba) XPS survey spectra for the surfaces of pristine silicone and IPN with and without SPMA, Bb) depth evaluation of N1s spectra with inset showing the constant intensity of nitrogen as the function of etching sequence of IPN, and Bc) colour change of cross-cut IPN after exposure to UV light. 

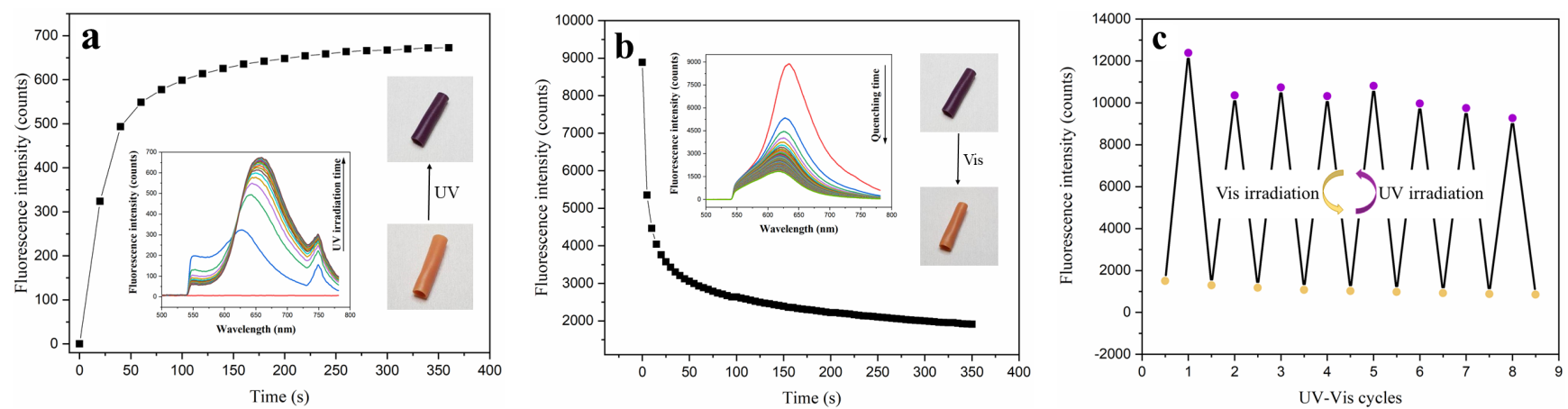

Figure 4. Time-dependent fluorescence emitted from poly(BMA-co-HEMA-co-SPMA) IPN with 8.5\% HEMA upon exposure to UV (a) and visible (b) light. The insets show the change in fluorescence spectra with increasing light exposure time and pictures of IPNs upon UV and visible light irradiation. (c) Monitoring of cyclic photo-isomerization effects on the fluorescence intensity of IPN.

Figure $4 \mathrm{~b}$ shows the fluorescence spectra of the reverse reaction induced by exposure to Vis light $(532 \mathrm{~nm})$ (spectra recorded every $5 \mathrm{~s}$ ). The fluorescence emission is efficiently decreased (quenched) by Vis light, reaching a photo-stationary state after 6 min and as shown in the photo inset of Figure 4b, the purple IPN returns to its initial colour. The photo-fatigue resistance and photo-switchability of the fabricated IPN was examined by alternating cycles of UV-Vis irradiation (Figure 4c). Each irradiation took 6 min and the fluorescence emission at $\lambda_{\max }$ under the excitation at $532 \mathrm{~nm}$ was immediately monitored after each irradiation. Figure $4 \mathrm{c}$ demonstrates that the switching back and forth between UV and Vis light irradiation could be repeated for at least eight cycles with a slight decrease in fluorescence recovery. The reversible isomerization supports that the SP/MC moiety in the IPN is fairly fatigueresistant as it only shows a slight photo-degradation after several cycles of UV-Vis irradiation.

3.4. Loading of IPNs with drug. IPNs were loaded with drugs by passive diffusion, placing them in a solvent containing the dissolved drug. To select a suitable loading solvent, it is crucial to consider two aspects. First, the applied solvent should only swell the guest polymer and not the silicone, to avoid drug loading into the silicone. Second, it is known that using polar surroundings, especially protic solvents, bias the chemical equilibrium of photoactive molecules towards the MC form, which helps with better drug loading. The equilibrium between $\mathrm{SP}$ and $\mathrm{MC}$ is controlled by Gibbs free energy. In the hydrophilic MC form, the zwitterionic structure allows electrostatic, polar-polar interactions and hydrogen bonding with the solvent molecules. The more polar the solvent is, the stronger the interactions, which moves the equilibrium toward the $\mathrm{MC}$ form ${ }^{74}$. In a polar solvent like ethanol, the energy level of MC is lower than that of SP, causing the isomerization of SP to MC to be thermodynamically spontaneous and irreversible (negative photochromism) at dark $(\Delta \mathrm{G}<0){ }^{74-76}$. Hence, by choosing a protic solvent, like ethanol, as the loading solvent, the hydrophobic SP moieties of the IPN isomerize to the hydrophilic $\mathrm{MC}$, giving rise to swelling of the guest polymer that allows diffusion of the drug into the IPN. The drug molecules are absorbed and adsorbed to the guest polymer and to the exposed interface between silicone and guest polymer inside the matrix. Using ethanol eliminates the need for UV irradiation to swell the samples in the loading step, which otherwise might lead to photo-fatigue of the IPNs.
3.5 Light-triggered release of DOX from the IPN. The results of DOX release with and without UV light irradiation is shown in Figure 5. It can be observed that the poly(BMA-coSPMA) IPN (Figure 5a) have much lower premature release compared with the poly(HEMA-co-PEGMEA-co-SPMA) IPN (Figure 5b), which is consistent with the values of work of adhesion of these two IPNs estimated by the model. As shown in Figure 5a, the cumulative release of DOX from the hydrophobic poly(BMA-co-SPMA) IPN upon UV exposure was almost 2.5 times higher than without UV light. This is attributed to the isomerization of SPMA to MCMA in the IPN. Except for the initial burst release, which may be due to DOX molecules on the surface of IPN, the drug was efficiently embedded inside the bulk of IPN, while its release could be triggered by light illumination. This burst release can be avoided by rinsing the IPN surface more thoroughly before starting the release test. For the more hydrophilic poly(HEMA-coPEGMEA-co-SPMA) IPN (Figure 5b), a substantial premature release was observed due to premature swelling of the guest polymer in water even without UV radiation. This was also indicated in water uptake measurements (Figure S5 in SI), showing that the water uptake of poly(HEMA-coPEGMEA-co-SPMA) IPN was comparatively high (around $23 \%$ ) even without UV on. When SPMA isomerizes to MCMA, the water uptake increases, which in turn increases the diffusion coefficient for DOX in the IPN due to less resistance. Another explanation could be related to the shift of the SPMA and MCMA chemical equilibrium. Through the polymerisation of HEMA with SPMA, the HEMA monomers surround the SPMA molecules and act as a protic environment, which means that hydrogen bonding between MCMA and hydroxyl groups of HEMA could inhibit the isomerizarion of MCMA back to SPMA at dark. Therefore, the guest polymer stays in its hydrophilic state, which causes diffusion of water and subsequent release of the drug. This explanation is consistent with previous results reported by others ${ }^{74-76}$, who demonstrated that SP has a negative photochromism in polar surroundings due to hydrogen bonding, especially in protic solvents. This explanation is further supported by the fact that the IPN with poly(HEMA-co-PEGMEA-co-SPMA) (Figure 2) has a more reddish taint compared to poly(BMA-coSPMA) at dark. The hydrophobic poly(BMA-co-SPMA) IPN showed however a very small accumulated release $(\sim 25 \mu \mathrm{g})$ in comparison to poly(HEMA-co-PEGMEA-co-SPMA) IPN 
(>3000 $\mu \mathrm{g}$ ). This could be due to that: (i) DOX, as a hydrophilic drug with a $\log \mathrm{P}$ value of -1.9 , has a lower loading efficiency in the hydrophobic poly(BMA-co-SPMA) IPN, and/or (ii) the low guest polymer content of $28 \%$ may not be
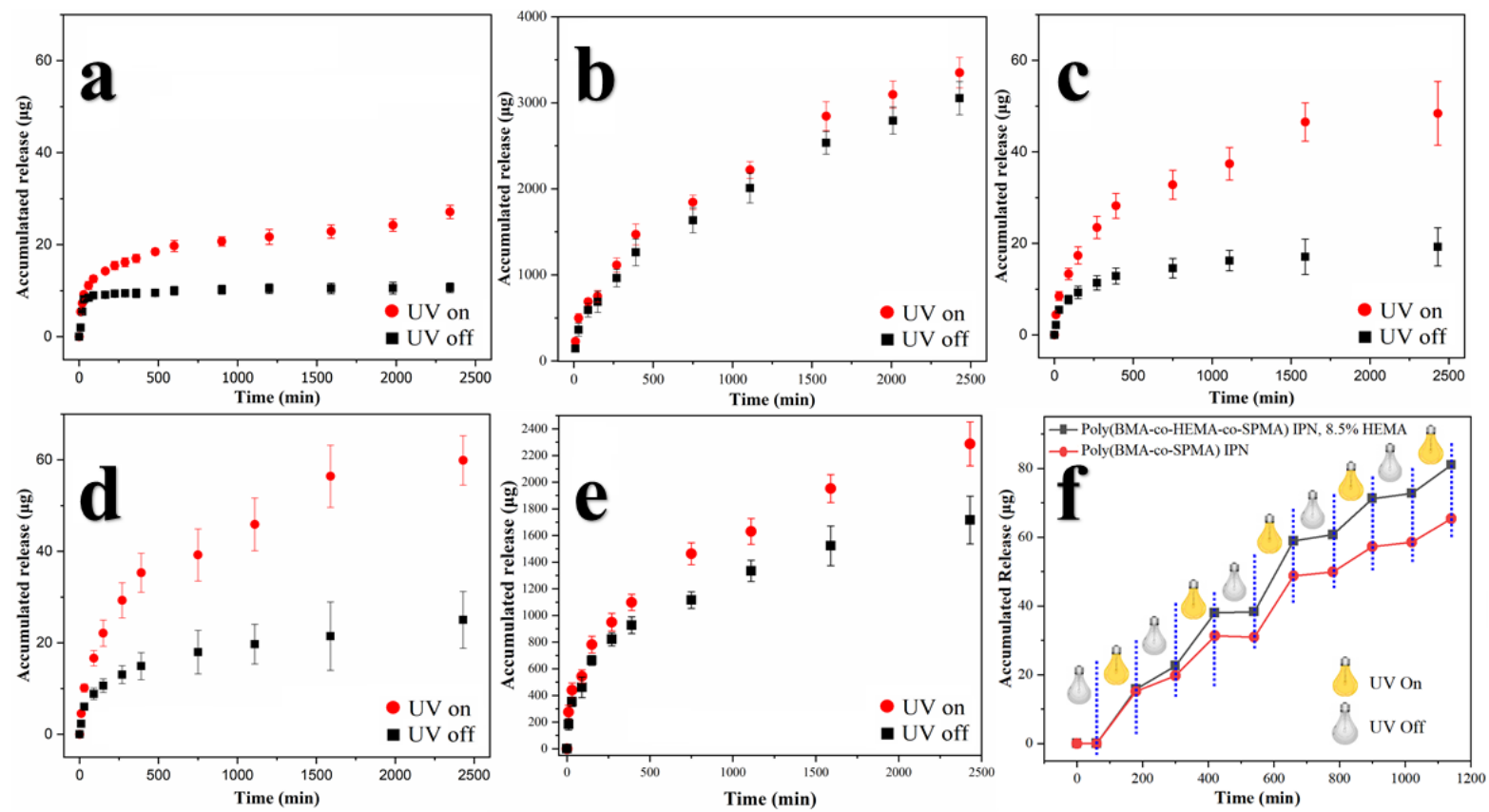

Figure 5. The release profile of DOX from: a) poly(BMA-co-SPMA), b) poly(HEMA-co-PEGMEA-co-SPMA), c) poly(BMA-co-HEMAco-SPMA) 8.5\% HEMA, d) poly(BMA-co-HEMA-co-SPMA) 12\% HEMA and e) poly(BMA-co-HEMA-co-SPMA) 24\% HEMA IPNs as a function of time. f) DOX released vs. time with UV light continuously switched on and off every two hours, comparing two of the IPNs. Data are presented for three identically treated IPNs as mean \pm standard deviation $(n=3)$.

enough to provide a sufficient surface connectivity between silicone and guest polymer. Silicone, which has a low glass transition temperature, might bleed to the surface and cover the guest polymer at the surface.

This can actually be seen in the inset of Figure $3 \mathrm{Bb}$, where the presence of $\mathrm{N}$-atoms (corresponding to the guest polymer) is lower in the outer layer of the IPN. When adding small amounts of HEMA ( $8.5 \%$ of the total guest polymer) to the monomer feed, the guest polymer content increased from $28 \%$ to $35 \%$, and the work of adhesion with DOX decreased, but was still relatively high (Table 2 ). The resulting poly(BMAco-HEMA-co-SPMA) IPN, which is slightly less hydrophobic than the poly(BMA-co-SPMA) IPN, showed triggered release of DOX and accumulated release of $\sim 50 \mu \mathrm{g}$ (Figure $5 \mathrm{c}$ ), which was an improvement compared to poly(BMA-coSPMA) IPN with $25 \mu \mathrm{g}$ release. As Figure $5 \mathrm{c}$ and d indicates, spiking the monomer feed with small amounts of HEMA (8.5 and $12 \%$ ) increases the accumulated release with UV on, and inhibits the premature release with UV off. However, by increasing the molar ratio of HEMA in the guest polymer to $24 \%$ the premature release was again significantly increased (Figure 5e), which is in line with the model-estimated work of adhesion values in Table 2 . The substantial premature release with 24\% HEMA can be a combined effect of high guest polymer content $(49 \% \mathrm{w} / \mathrm{w})$ and small work of adhesion with DOX. These findings show that the drug loading and accumulated release can be adjusted by changing the co-polymers in the system. Figure $5 \mathrm{f}$ shows the release of DOX from poly(BMA-co-SPMA) IPN and poly(BMA-co-HEMA-co-
SPMA) IPNs when the light is repeatedly switched on and off every two hours. It can be seen that the release rate is increased when the light is switched on and halted or decreased when light is switched off. This reversible feature is rather unique for a triggered DDS. These on-off experiments demonstrated that the release of a drug can be triggered and controlled by light. It was concluded that the guest polymer composition, especially the hydrophilicity of the guest polymer, has a huge impact on the work of adhesion, premature release and whether the release can be triggered. Moreover, that the developed thermodynamic model is adequate to design and optimize the guest polymer composition. Considering the model-estimated work of adhesion and the release experiments, the poly(BMA-co-HEMA-co-SPMA) IPN with $8.5 \%$ HEMA was selected as the optimal IPN since it showed a limited premature release and the highest accumulated release compared to the other two investigated IPNs. This IPN was further used for comparing release profiles of five different drugs with different $\log \mathrm{P}$ values.

3.6. Evaluation of the effect of drug lipophilicity on the triggered release using the thermodynamic model.

Considering eq. (2), the simplified form of eq. (7), the hydrophobic-to-hydrophilic switch (SPMA to MCMA) should lead to a change of the HSP of the polymers, and hence the values of $\gamma_{\mathrm{PD}}$ and $\gamma_{\mathrm{PW}}$ in eq. (2) should change. However, whether or not the light-induced SPMA to MCMA switch can overcome the work of adhesion depends on the interfacial surface tension between the drug and polymer, and the way it changes upon UV exposure. In order to investigate the effect 
of the lipophilicity ( $\log \mathrm{P}$ value) of a drug on its release behaviour, release studies were performed on poly(BMA-coHEMA-co-SPMA) IPNs with $8.5 \%$ HEMA for a series of drugs with different $\log \mathrm{P}$ values; dopamine, levopoda (L- dopa), prednisone and curcumin (cf. Table 1), and the resulting release profiles, fitted to the power law equation, are shown in Figure 6. Figure 5c for DOX can be directly compared with the release profiles in Figure 6.
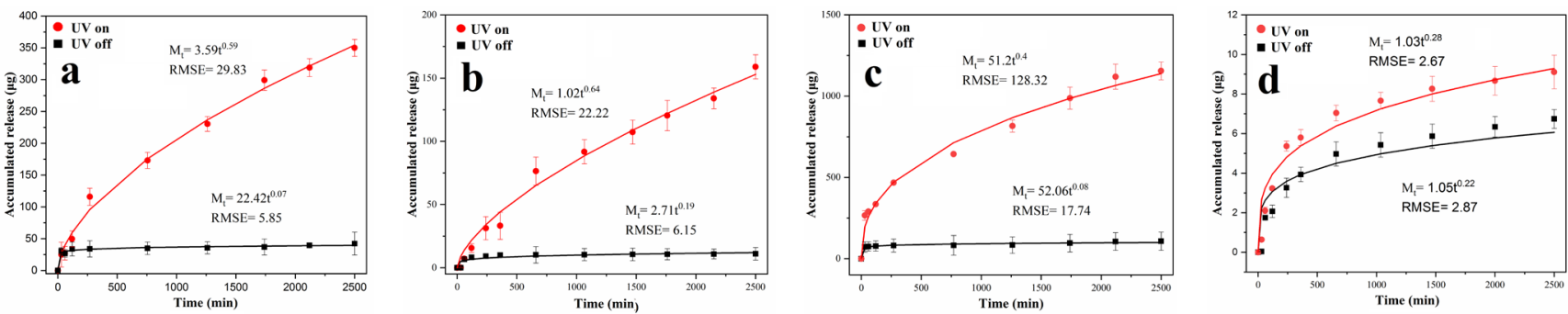

Figure 6. Light-triggered release profiles of: a) dopamine, b) L-dopa, c) prednisone, and d) curcumin from the poly (BMA-co-HEMA-coSP) IPN with $8.5 \%$ HEMA. The data fitted to power law equation describes the release. Data are presented for three identically treated IPNs as mean \pm standard deviation $(\mathrm{n}=3)$.

One of the most applied models to describe controlled release of drugs from polymeric devices is the semi-empirical power law equation, $M_{t}=K t^{n}$, developed by Ritger and Peppas ${ }^{77}$ where $M_{t}$ is the accumulated release of drug at time $t, K$ is a constant incorporating structural and geometric characteristics, and $n$ is the diffusional exponent, which is dependent on the geometry of the sample as well as the physical mechanism for release. When comparing the release behavior with and without UV exposure, considering that the geometry of the samples is the same, the difference between diffusional exponents determine the change in the mechanism of release. Drug transport from a slab geometry is categorized as Fickan diffusion $(\mathrm{n}=0.5)$, case II transport $(\mathrm{n}=1)$, non-Fickian or anomalous transport $(0.5<\mathrm{n}<1)$ and super case II transport $(n>1){ }^{77}$. The release of dopamine, L-dopa, prednisone and curcumin from the photoresponsive IPN is fitted to the Power law equation and shown in Figure. As seen, the model describes the data of the release quite well with acceptable RMSE which are shown in Figure 6. DOX, dopamine, L-dopa and prednisone all show triggered release, i.e. the release is triggered upon exposure to UV light with a very low premature release. Curcumin has a comparatively high premature release (UV off), however, the triggered release of curcumin (UV on) is very low (only a few $\mu \mathrm{g}$ ) compared to the other drugs. The reason could be its low water solubility of $5.75 \mu \mathrm{g} / \mathrm{mL}$ (Table 1), i.e., only a few curcuminmolecules can be dissolved in the release media, hence forcing the chemical equilibrium towards adsorption. Due to the relatively small water solubility, the release rate is expected to be low as the concentration of dissolved and desorbed curcumin, and hence the driving force for diffusion would be very small. In order to understand the system and how the hydrophobic-to-hydrophilic switch of SPMA can overcome the work of adhesion between drug and polymer, the developed thermodynamic model (eq. (7)) was used.

3.6.1. Comparison of experimental results with the thermodynamic model. The HSP for the spiropyran monomer before and after isomerisation (SPMA and MCMA) was estimated by the group contribution method. The existing groups in SPMA and MCMA and their contribution to HSP is presented in Table S4 and Table S5 in SI. By inserting HSP of the drugs ${ }^{78-80}$ (cf. Table 1), monomers (SPMA and
MCMA) and solvent (water) in eqn. (7), the work of adhesion before and after exposing the IPNs to light was calculated and the results presented in Figure 7. This figure combines the $\log \mathrm{P}$ value of the tested drugs with the work of adhesion before and after exposure to UV light, calculated from eq (9) and experimental release studies presented in Figure $5 \mathrm{c}$ and Figure 6 . The work of adhesion is read on the left y-axis. The "triggered release $(T) \%$ " (green bars) is a measure for how much of the release is caused by the trigger and is calculated from eq. (9). The green bars are read on the right y-axis.

$$
T(\%)=\frac{\text { release }_{u v o n}-\text { release }_{u v \text { off }}}{\text { release }_{u v \text { on }}} \times 100 \%
$$

As can be seen from Figure 7, the work of adhesion between the SPMA and all five drugs before UV (black squares), changed to smaller values after UV exposure (red circles), which is consistent with the experimental results, displaying triggered release upon light exposure, especially for dopamine, L-dopa and prednisone with around 80-90\% triggered release. This makes sense because the IPN becomes more hydrophilic after UV exposure due to the transition from SPMA to MCMA. Hence, it becomes more energetically favourable for water to bind to the polymer than it is for the drug to bind to the polymer. For DOX ( $\log \mathrm{P}$ value of -1.9), we see that the model-estimated work of adhesion is only slightly changed after UV exposure, which explain why the experimental release results show less triggered effect for DOX. 


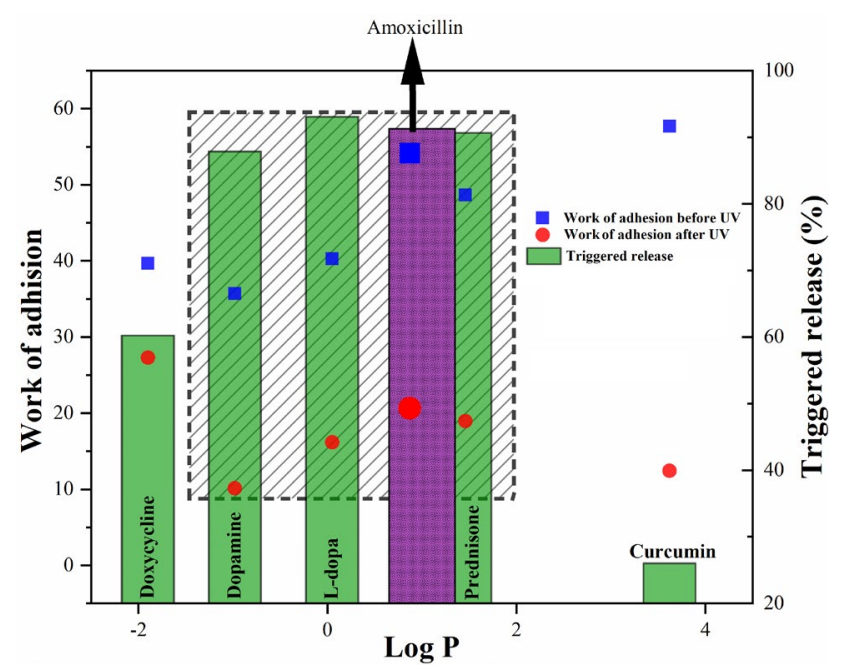

Figure 7. The relationship between the $\log \mathrm{P}$ values of drugs ( $\mathrm{x}-$ axis), the work of adhesion before (black squares) and after (red circles) UV exposure, calculated using the thermodynamic model (left y-axis), and the triggered release, derived from experimental results (green bars, y-axis). The shaded area indicates within which area the studied drugs can be triggered from the poly(BMA-co-HEMA-co-SPMA) IPN with $8.5 \%$ HEMA. The grey bar shows the results for amoxicillin.

However, there is an enhanced release rate after UV exposure with a triggered release of around $60 \%$. If we compare the accumulated release of DOX from the hydrophilic poly(HEMA-co-PEGMEA-co-SPMA) with the partially hydrophobic poly(BMA-co-HEMA-co-SPMA, $8.5 \%$ HEMA) (cf. Figure 5b and c), the hydrophilic IPN released around $3000 \mu \mathrm{g}$ DOX whereas the partially hydrophobic IPN released only around $50 \mu \mathrm{g}$. One possible explanation could be a poor loading into the hydrophobic IPN, i.e. DOX being so hydrophilic that it prefers to stay in the loading media instead of adsorbing/absorbing to the guest polymer. Considering the large difference in the work of adhesion for curcumin before and after UV exposure (Figure 7), the model tells us that we should have seen a high triggered release, however, it was only $26 \%$ (Figure 7 ) with an accumulated release of less than $10 \mu \mathrm{g}$ (Figure $6 \mathrm{~d}$ ) both before and after UV exposure. This is likely due to the combination of the low water solubility, as discussed above, and that it adsorbs to or even absorbs into the silicone matrix due to its high $\log \mathrm{P}$ value (3.62). Once it is desorbed, the difference in concentration of dissolved curcumin inside and outside the IPN is very small and hence the driving force for diffusion out of the IPN is likewise also very small. It should however be noted that the values shown in Figure 7 are estimated based entirely on the work of adhesion between the drug and the SPMA moieties of the guest polymer, since they are key for the triggered release.

These findings offer compelling evidence for that drugs with $\log \mathrm{P}$ - and work of adhesion values, in the region specified by the shaded area in Figure 7, should have triggered release with the developed IPNs, whereas release of drugs with higher or lower $\log \mathrm{P}$ values cannot be triggered. Such drugs will have less accumulated release and higher premature release, respectively. To finally test this assumption, a new drug with a $\log \mathrm{P}$ value within the shaded area was chosen at random and its triggered release evaluated. This compound was amoxicillin with a $\log \mathrm{P}$ value of 0.47 and a water solubility of $2.7 \mathrm{mg} / \mathrm{ml}$. The model-estimated work of adhesion before and after UV exposure was calculated to be 20.63 and $54.17 \mathrm{mN} / \mathrm{m}$, respectively, both values within in the shaded area (Figure 7) and led to a triggered release of $89 \%$.

3.7. Biocompatibility test (Cell viability and immunocytochemistry). An appropriate drug delivery system, for use in medical devices or in tissue engineering applications, has to be biocompatible. Here, we used human neural stem cells (hNSCs) to test the potential of the optimized IPNs as a biocompatible substrate for cell-related applications. In contrast to more conventional and robust cell lines, hNSCs are highly sensitive to environmental perturbation and as such provide an optimal in vitro system for testing of new substrates ${ }^{81}$. Furthermore, these cells can be differentiated into dopaminergic neurons used in cell replacement therapy for Parkinson's disease where IPNs could be utilized to supply cells with L-dopa, a precursor needed for dopamine production of these neurons. Cell survival on any given material construct is the first crude indicator of the construct's biocompatibility. Therefore, cell viability and survival of hNSCs was assessed through live/dead staining 24 hours after culturing on the IPNs in growth medium. The staining reveals that live cells are uniformly distributed on the IPNs surface with spindle-like morphology (see Figure 8a). High viability (more than $98 \%$, Figure 8 b) together with healthy morphology of hNSCs confirms that the IPNs do not have any significant detrimental effects on stem cell survival, adhesion, and spreading. Exposure of the IPN to UV did not alter significantly viability, adhesion, and morphology of hNSCs (Figure 8c,d and Figure S7c,d). Then, to show that the photoactive IPN can be used as a substrate for the generation of stem-cell derived dopaminergic neurons, a highly delicate process, hNSCs were differentiated on the IPNs at the given experimental differentiation conditions (Figure 8e and Figure S7e). After 10 days, immunocytochemistry was performed to assess the efficacy of the differentiation. Differentiated hNSCs were immunostained for ß-tubulin III (a biomarker that identifies neuronal cytoskeleton proteins), tyrosine hydroxylase ( $\mathrm{TH}$, the rate-limiting enzyme in dopamine synthesis, identifying dopaminergic neurons) and the cell nuclei counterstained with Hoechst (a blue fluorescent dye that binds to the DNA of the nuclei). The obtained fluorescent images show that no toxic chemical able to hinder the differentiation process leaked from the IPNs into the media. Figures 8e and S7e show a high expression of the neuronal marker $\beta$-tubulin III, indicating that neurons were successfully generated on both IPNs without any observable issues with neuronal morphology. Moreover, cells did not make aggregates but formed a dense network of projections that covered the whole IPN surface. At the same time, dopaminergic neurons were successfully generated on the IPNs, as indicated by the presence of TH. Overall the results show that these photoresponsive IPNs enable successful hNSC differentiation into neurons with characteristics in line with previously published results using conventional polystyrene tissue culture well plates ${ }^{82,83}$. 

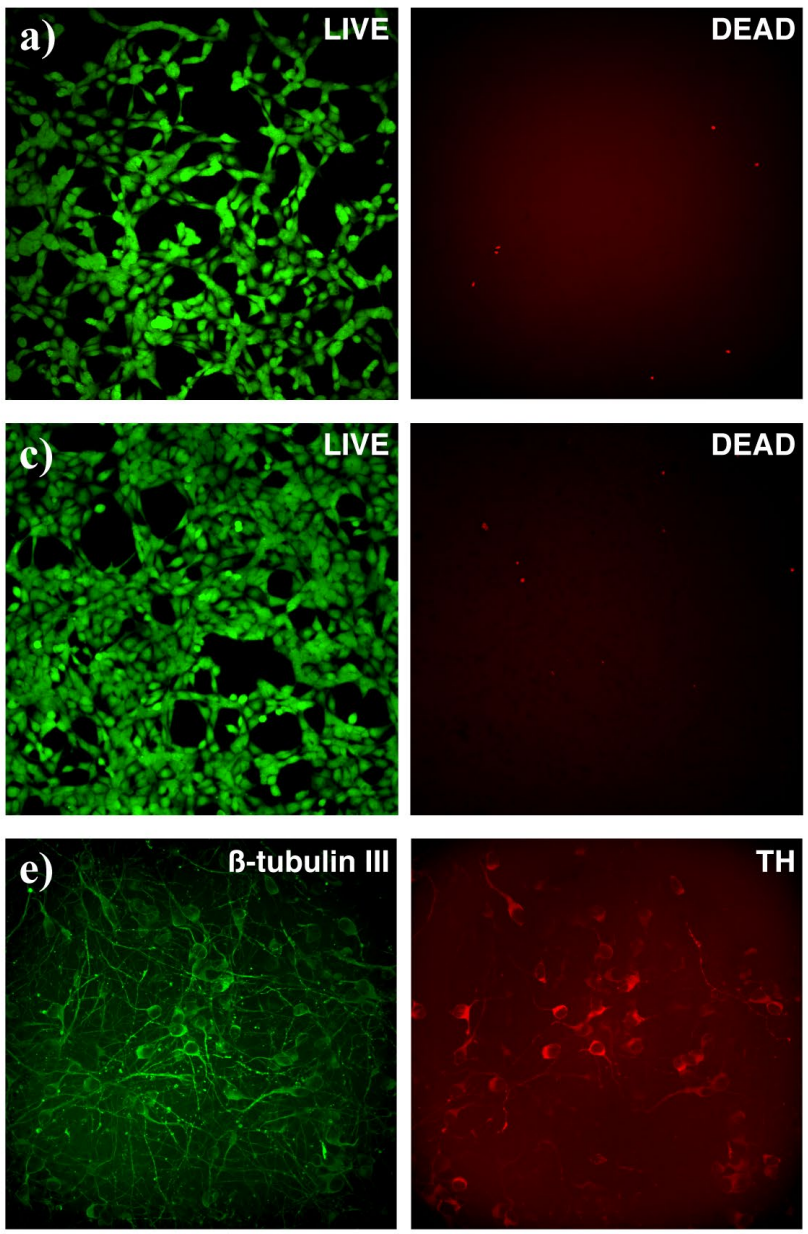
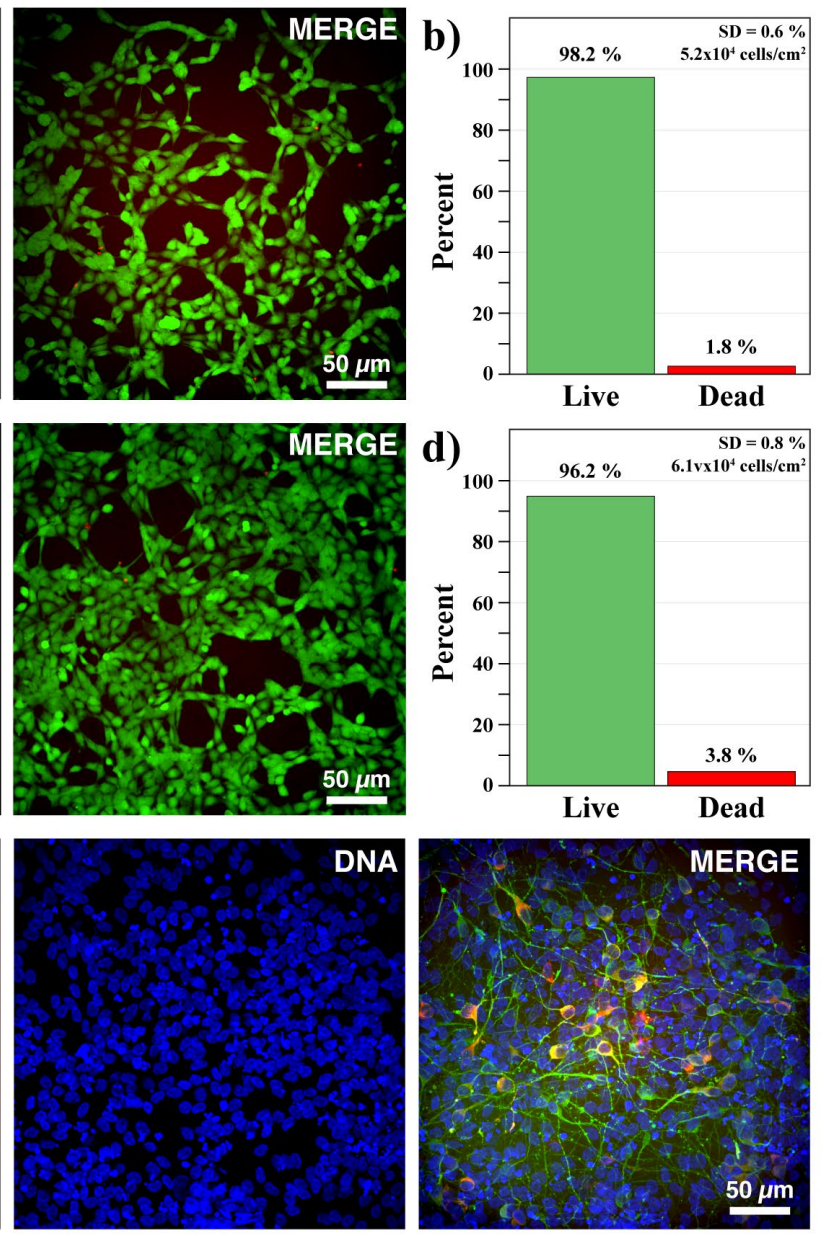

Figure 8. Viability of hNSCs and their differentiation into dopaminergic neurons on poly(BMA-co-HEMA-co-SP) IPN, 8.5\% HEMA: a) Fluorescence confocal images of cells stained with live (Calcein AM) and dead (EthD-1) stain 24 hours after seeding without UV exposure. b) Statistical analysis of cell viability $(\mathrm{n}=3)$. c) Live/dead staining of cells on UV-exposed IPN. d) Respective stastistical analysis of cell viability $(n=3)$. e) Maximum intensity projections of fluorescence confocal images taken from $150 \mu$ m thick optical section. Immunocytochemistry shows an even coverage of the IPN by the differentiated neurons: $\beta$ - III tubulin (green); TH (red); nuclei (blue); merge (green, red, blue).

\section{CONCLUSION}

Light-triggered DDS based on IPNs were successfully achieved using the $\mathrm{scCO}_{2}$ technology. The method of developing silicone-based IPN has some advantages in terms of versatility in that the geometry and dimension of the final constructs can easily be altered in regards to the shape of the silicone used. The developed photo-responsive IPN brings about a new concept of light-triggered DDS, exploiting the change in intermolecular interactions (work of adhesion) between polymer, drug and solvent, as the hydrophobic SP moiety in the polymer transform into the hydrophilic MC, triggering drug release without degradation and disruption of the DDS. The developed DDS has the property that the release of drug can be repeatedly switched on and off, simply by switching the light on and off.

The theory of drug release from the carrier using the concept of work of adhesion and surface chemistry based on HSP was investigated. It was shown that the developed thermodynamic model was a new tool for designing, developing and optimizing the IPN polymer composition to tailor and minimize the premature release. Comparing the results obtained from the experimental results and the work of adhesions estimated by the developed thermodynamic model shows that the model is useful for explaining the triggered release behaviour and estimating whether exposure to UV light can trigger the release of a given drug from a given IPN in a given solvent, and it was been. The model, however, has its limits. It cannot provide information about the solubility of the drugs in the loading media.

\section{ASSOCIATED CONTENT}

\section{Supporting Information}

The Supporting Information is available free of charge on the ACS Publications website.

H-NMR spectra of SPOH and SPMA (S1), the composition of the monomers solutions for fabricating photo-responsive IPNs with different guest polymers (S2), full derivation of the development of the thermodynamic model (S3), estimation of HSP of monomers and copolymers as the guest polymers of IPNs (S4), ATRFTIR spectra of poly(BMA-co-SPMA) IPN and poly(HEMA-coPEGMEA-co-SPMA) IPN (S5), the water uptake measurement of IPNs and its result (S6) calculation of the HSP of SPMA and MCMA using group contribution method and the work of adhesion (S7), Cell viability and immunocytochemistry of hNSCs 
cells on of the IPN (S8), Two-photon NIR excitation of IPNs (S9), Photochromic properties of SPOH (S10). (PDF).

\section{AUTHOR INFORMATION}

\section{Corresponding Author}

*corresponding authors: jemn@dtu.dk; ma@biomodics.com

\section{Author Contributions}

The manuscript was written through contributions of all authors. / All authors have given approval to the final version of the manuscript.

\section{ACKNOWLEDGMENT}

This project was funded by the European Union Horizon 2020 Programme (H2020-MSCA-ITN-2016) under the Marie Skłodowska-Curie Innovative Training Network, project Training4CRM with Grant Agreement No. 722779.

\section{REFERENCES}

(1) Kaurav, H.; Kapoor, D. N. Implantable Systems for Drug Delivery to the Brain. Ther. Deliv. 2017, 8 (12), 1097-1107. https://doi.org/10.4155/tde-2017-0082.

(2) Chandy, T. Biocompatibility of Materials and Its Relevance to Drug Delivery and Tissue Engineering. In Biointegration of Medical Implant Materials; Elsevier, 2020; pp 297-331. https://doi.org/10.1016/B978-0-08-102680-9.00012-3.

(3) Ruskowitz, E. R.; DeForest, C. A. Photoresponsive Biomaterials for Targeted Drug Delivery and 4D Cell Culture. Nat. Rev. Mater. 2018, 3 (2), 17087. https://doi.org/10.1038/natrevmats.2017.87.

(4) Mura, S.; Nicolas, J.; Couvreur, P. Stimuli-Responsive Nanocarriers for Drug Delivery. Nat. Mater. 2013, 12 (11), 991-1003. https://doi.org/10.1038/nmat3776.

(5) Kelley, E. G.; Albert, J. N. L.; Sullivan, M. O.; Epps, III, T. H. Stimuli-Responsive Copolymer Solution and Surface Assemblies for Biomedical Applications. Chem. Soc. Rev. 2013, 42 (17), 7057. https://doi.org/10.1039/c3cs35512h

(6) Linsley, C. S.; Wu, B. M. Recent Advances in LightResponsive on-Demand Drug-Delivery Systems. Therapeutic Delivery. Future Medicine Ltd. February 1, 2017, pp 89-107. https://doi.org/10.4155/tde-2016-0060.

(7) Mura, S.; Nicolas, J.; Couvreur, P. Stimuli-Responsive Nanocarriers for Drug Delivery. Nat. Mater. 2013, 12 (11), 991-1003. https://doi.org/10.1038/nmat3776.

(8) Sortino, S. Photoactivated Nanomaterials for Biomedical Release Applications. J. Mater. Chem. 2012, 22 (2), 301-318. https://doi.org/10.1039/C1JM13288A.

(9) Rwei, A. Y.; Wang, W.; Kohane, D. S. Photoresponsive Nanoparticles for Drug Delivery. Nano Today 2015, 10 (4), 451-467. https://doi.org/10.1016/j.nantod.2015.06.004.

(10) Olejniczak, J.; Carling, C.-J.; Almutairi, A. Photocontrolled Release Using One-Photon Absorption of Visible or NIR Light. J. Control. Release 2015, 219, 18-30. https://doi.org/10.1016/j.jconrel.2015.09.030.

(11) Lu, J.; Choi, E.; Tamanoi, F.; Zink, J. I. Light-Activated Nanoimpeller-Controlled Drug Release in Cancer Cells. Small 2008, 4 (4), 421-426. https://doi.org/10.1002/smll.200700903.

(12) He, D.; He, X.; Wang, K.; Cao, J.; Zhao, Y. A LightResponsive Reversible Molecule-Gated System Using Thymine-Modified Mesoporous Silica Nanoparticles. Langmuir 2012, $28 \quad$ (8), 4003-4008. https://doi.org/10.1021/1a2047504.

(13) Agasti, S. S.; Chompoosor, A.; You, C. C.; Ghosh, P.; Kim, C. K.; Rotello, V. M. Photoregulated Release of Caged Anticancer Drugs from Gold Nanoparticles. J. Am. Chem. Soc. 2009, 131 (16), 5728-5729. https://doi.org/10.1021/ja900591t.

(14) Tong, R.; Hemmati, H. D.; Langer, R.; Kohane, D. S. Photoswitchable Nanoparticles for Triggered Tissue Penetration and Drug Delivery. J. Am. Chem. Soc. 2012, 134 (21), 8848-8855. https://doi.org/10.1021/ja211888a.
Pavlukhina, S.; Sukhishvili, S. Polymer Assemblies for
Controlled Delivery of Bioactive Molecules from Surfaces. Adv. Drug Deliv. Rev. 2011, 63 (9), 822-836. https://doi.org/10.1016/j.addr.2011.03.017.

Volodkin, D. V; Madaboosi, N.; Blacklock, J.; Skirtach, A. G. Surface-Supported Multilayers Decorated with Bio-Active Material Aimed at Light-Triggered Drug Delivery $\dagger$. Phys. Chem. Chem. Phys 2009, 25 (24), 6306-6311. https://doi.org/10.1021/la9015433.

Fomina, N.; Sankaranarayanan, J.; Almutairi, A Photochemical Mechanisms of Light-Triggered Release from Nanocarriers. Adv. Drug Deliv. Rev. 2012, 64 (11), 1005-1020. https://doi.org/10.1016/j.addr.2012.02.006.

Son, S.; Shin, E.; Kim, B. S. Light-Responsive Micelles of Spiropyran Initiated Hyperbranched Polyglycerol for Smart Drug Delivery. Biomacromolecules 2014, 15 (2), 628-634. https://doi.org/10.1021/bm401670t.

(19) Minkin, V. I. Photo-, Thermo-, Solvato-, and Electrochromic Spiroheterocyclic Compounds. Chem. Rev. 2004, 104 (5), 2751-2776. https://doi.org/10.1021/cr020088u.

(20) Berkovic, G.; Krongauz, V.; Weiss, V. Spiropyrans and Spirooxazines for Memories and Switches. Chem. Rev. 2000, 100 (5), 1741-1753. https://doi.org/10.1021/cr9800715.

(21) Cardano, F.; Del Canto, E.; Giordani, S. Spiropyrans for LightControlled Drug Delivery. Dalt. Trans. 2019, 48 (41), 1553715544. https://doi.org/10.1039/c9dt02092f.

(22) Klajn, R. Spiropyran-Based Dynamic Materials. Chem. Soc. Rev. 2014, $43 \quad$ (1), 148-184 https://doi.org/10.1039/C3CS60181A.

(23) Lenoble, C.; Becker, R. S. Photophysics, Photochemistry, Kinetics, and Mechanism of the Photochromism of 6'Nitroindolinospiropyran. J. Phys. Chem. 1986, 90 (1), 62-65. https://doi.org/10.1021/j100273a015.

(24) Ventura, C.; Thornton, P.; Giordani, S.; Heise, A. Synthesis and Photochemical Properties of Spiropyran Graft and Star Polymers Obtained by "click" Chemistry. Polym. Chem. 2014, 5 (21), 6318-6324. https://doi.org/10.1039/c4py00778f.

(25) Canto, E. Del; Natali, M.; Movia, D.; Giordani, S. PhotoControlled Release of Zinc Metal Ions by Spiropyran Receptors Anchored to Single-Walled Carbon Nanotubes. Phys. Chem Chem. Phys. 2012, 14 (17), 6034-6043. https://doi.org/10.1039/c2cp40275k.

(26) Koçer, A.; Walko, M.; Meijberg, W.; Feringa, B. L. A LightActuated Nanovalve Derived from a Channel Protein. Science (80-. ). 2005, $309 \quad$ (5735), 755-758. https://doi.org/10.1126/science.1114760.

(27) Ma, T.; Walko, M.; Lepoitevin, M.; Janot, J. M.; Balanzat, E.; Kocer, A.; Balme, S. Combining Light-Gated and PHResponsive Nanopore Based on PEG-Spiropyran Functionalization. Adv. Mater. Interfaces 2018, 5 (2), 1701051. https://doi.org/10.1002/admi.201701051.

(28) Ali, A. A.; Kharbash, R.; Kim, Y. Chemo- and Biosensing Applications of Spiropyran and Its Derivatives - A Review. Anal. Chim. $\quad$ Acta https://doi.org/10.1016/j.aca.2020.01.057.

(29) Yang, Z.; Zou, H.; Liu, H.; Xu, W.; Zhang, L. Self-Assembly and Drug Release Control of Dual-Responsive Copolymers Based on Oligo(Ethylene Glycol)Methyl Ether Methacrylate and Spiropyran. Iran. Polym. J. 2019, 28 (1), 39-49. https://doi.org/10.1007/s13726-018-0677-7.

(30) Chen, J.; Zhong, W.; Tang, Y.; Wu, Z.; Li, Y.; Yi, P.; Jiang, J. Amphiphilic BODIPY-Based Photoswitchable Fluorescent Polymeric Nanoparticles for Rewritable Patterning and DualColor Cell Imaging. Macromolecules 2015, 48 (11), 35003508. https://doi.org/10.1021/acs.macromol.5b00667.

(31) Shao, N.; Zhang, Y.; Cheung, S.; Yang, R.; Chan, W.; Mo, T.; Li, K.; Liu, F. Copper Ion-Selective Fluorescent Sensor Based on the Inner Filter Effect Using a Spiropyran Derivative. Anal. $\begin{array}{lllll}\text { Chem. } & 2005, & 77 & \text { (22), 7294-7303. }\end{array}$ https://doi.org/10.1021/ac051010r.

(32) Xie, X.; Mistlberger, G.; Bakker, E. Reversible Photodynamic Chloride-Selective Sensor Based on Photochromic Spiropyran. J. Am. Chem. Soc. 2012, 134 (41), 16929-16932. https://doi.org/10.1021/ja307037z.

(33) Rapoport, N. Physical Stimuli-Responsive Polymeric Micelles for Anti-Cancer Drug Delivery. Prog. Polym. Sci. 2007, 32 (8- 
Bobrovsky, A. Y.; Boiko, N. I.; Shibaev, V. P. Photosensitive Cholesteric Copolymers with Spiropyran-Containing Side Groups: Novel Materials for Optical Data Recording. Adv. Mater. 1999, $11 \quad$ (12), 1025-1028. https://doi.org/10.1002/(SICI)15214095(199908)11:12<1025::AID-ADMA1025>3.0.CO;2-4.

(35) Natarajan, L. V.; Tondiglia, V.; Bunning, T. J.; Crane, R. L.; Adams, W. W. Liquid Crystalline Siloxanes Containing Spiropyran Chromophores as Reversible Optical Data Storage Materials. Adv. Mater. Opt. Electron. 1992, 1 (6), 293-297. https://doi.org/10.1002/amo.860010606.

(36) Kawata, S.; Kawata, Y. Three-Dimensional Optical Data Storage Using Photochromic Materials. Chem. Rev. 2000, 100 (5), 1777-1788. https://doi.org/10.1021/cr980073p.

(37) Aibani, N.; da Costa, P. F.; Masterson, J.; Marino, N.; Raymo, F. M.; Callan, J.; Callan, B. The Integration of Triggered Drug Delivery with Real Time Quantification Using FRET; Creating a Super 'Smart' Drug Delivery System. J. Control. Release 2017, 264 ,

136-144. https://doi.org/10.1016/j.jconrel.2017.08.013.

(38) Chen, C.-J.; Jin, Q.; Liu, G.-Y.; Li, D.-D.; Wang, J.-L.; Ji, J. Reversibly Light-Responsive Micelles Constructed via a Simple Modification of Hyperbranched Polymers with Chromophores. Polymer (Guildf). 2012, 53 (17), 3695-3703. https://doi.org/10.1016/j.polymer.2012.06.024.

(39) Chen, L.; Wang, W.; Su, B.; Wen, Y.; Li, C.; Zhou, Y.; Li, M.; Shi, X.; Du, H.; Song, Y.; Jiang, L. A Light-Responsive Release Platform by Controlling the Wetting Behavior of Hydrophobic Surface. ACS Nano 2014, 8 (1), 744-751. https://doi.org/10.1021/nn405398d.

(40) Petriashvili, G.; Devadze, L.; Zurabishvili, T.; Sepashvili, N.; Chubinidze, K. Light Controlled Drug Delivery Containers Based on Spiropyran Doped Liquid Crystal Micro Spheres. Biomed. Opt. Express 2016, $7 \quad$ (2), 442. https://doi.org/10.1364/boe.7.000442.

(41) Cao, Z.; Wang, G.; Chen, Y.; Liang, F.; Yang, Z. LightTriggered Responsive Janus Composite Nanosheets. Macromolecules 2015, 48 (19), 7256-7261. https://doi.org/10.1021/acs.macromol.5b01257.

(42) Liu, C.; Zhang, Y.; Liu, M.; Chen, Z.; Lin, Y.; Li, W.; Cao, F.; Liu, Z.; Ren, J.; Qu, X. A NIR-Controlled Cage Mimicking System for Hydrophobic Drug Mediated Cancer Therapy. Biomaterials 2017, 139, 151-162. https://doi.org/10.1016/j.biomaterials.2017.06.008.

(43) Xing, Q.; Li, N.; Chen, D.; Sha, W.; Jiao, Y.; Qi, X.; Xu, Q.; $\mathrm{Lu}$, J. Light-Responsive Amphiphilic Copolymer Coated Nanoparticles as Nanocarriers and Real-Time Monitors for Controlled Drug Release. J. Mater. Chem. B 2014, 2 (9), 1182 1189. https://doi.org/10.1039/c3tb21269f.

(44) Aznar, E.; Casasús, R.; García-Acosta, B.; Dolores Marcos, M.; Martínez-Máñez, R.; Sancenón, F.; Soto, J.; Amorós, P. Photochemical and Chemical Two-Channel Control of Functional Nanogated Hybrid Architectures. Adv. Mater. 2007, 19 (17), 2228-2231. https://doi.org/10.1002/adma.200601958.

(45) Weissleder, R. A Clearer Vision for in Vivo Imaging: Progress Continues in the Development of Smaller, More Penetrable Probes for Biological Imaging. Nat. Biotechnol. 2001, 19 (4), 316-317. https://doi.org/10.1038/86684.

(46) Cho, H. J.; Chung, M.; Shim, M. S. Engineered PhotoResponsive Materials for near-Infrared-Triggered Drug Delivery. Journal of Industrial and Engineering Chemistry. November 2015, pp 15-25. https://doi.org/10.1016/j.jiec.2015.07.016.

(47) Schumers, J. M.; Fustin, C. A.; Gohy, J. F. Light-Responsive Block Copolymers. Macromol. Rapid Commun. 2010, 31 (18), 1588-1607. https://doi.org/10.1002/marc.201000108.

(48) Kim, A.; Zhou, J.; Samaddar, S.; Song, S. H.; Elzey, B. D.; Thompson, D. H.; Ziaie, B. An Implantable UltrasonicallyPowered Micro-Light-Source (MLight) for Photodynamic Therapy. Sci. Rep. 2019, $9 \quad$ (1), 1395. https://doi.org/10.1038/s41598-019-38554-2.

(49) Klein, K.; Grønnemose, R. B.; Alm, M.; Brinch, K. S.; Kolmos, H. J.; Andersen, T. E. Controlled Release of Plectasin NZ2114 from a Hybrid Silicone-Hydrogel Material for Inhibition of Staphylococcus Aureus Biofilm. Antimicrob. Agents Chemother. 2017, 61 (7). https://doi.org/10.1128/AAC.0060417.

(50) Stenger, M.; Klein, K.; Grønnemose, R. B.; Klitgaard, J. K.; Kolmos, H. J.; Lindholt, J. S.; Alm, M.; Thomsen, P.; Andersen, T. E. Co-Release of Dicloxacillin and Thioridazine from Catheter Material Containing an Interpenetrating Polymer Network for Inhibiting Device-Associated Staphylococcus Aureus Infection. J. Control. Release 2016, 241, 125-134. https://doi.org/10.1016/j.jconrel.2016.09.018.

(51) Riber, L.; Burmølle, M.; Alm, M.; Milani, S. M.; Thomsen, P.; Hansen, L. H.; Sørensen, S. J. Enhanced Plasmid Loss in Bacterial Populations Exposed to the Antimicrobial Compound Irgasan Delivered from Interpenetrating Polymer Network Silicone Hydrogels. Plasmid 2016, 87-88, 72-78. https://doi.org/10.1016/j.plasmid.2016.10.001.

(52) Ramstedt, M.; Ribeiro, I. A. C.; Bujdakova, H.; Mergulhão, F. J. M.; Jordao, L.; Thomsen, P.; Alm, M.; Burmølle, M.; Vladkova, T.; Can, F.; Reches, M.; Riool, M.; Barros, A.; Reis, R. L.; Meaurio, E.; Kikhney, J.; Moter, A.; Zaat, S. A. J.; Sjollema, J. Evaluating Efficacy of Antimicrobial and Antifouling Materials for Urinary Tract Medical Devices: Challenges and Recommendations. Macromolecular Bioscience. May 18, 2019, p 1800384. https://doi.org/10.1002/mabi.201800384.

(53) Mohanty, S.; Alm, M.; Hemmingsen, M.; Dolatshahi-Pirouz, A.; Trifol, J.; Thomsen, P.; Dufva, M.; Wolff, A.; Emnéus, J. 3D Printed Silicone-Hydrogel Scaffold with Enhanced Physicochemical Properties. Biomacromolecules 2016, 17 (4), 1321-1329. https://doi.org/10.1021/acs.biomac.5b01722.

(54) Steffensen, S. L.; Vestergaard, M. H.; Møller, E. H.; Groenning, M.; Alm, M.; Franzyk, H.; Nielsen, H. M. Soft Hydrogels Interpenetrating Silicone - A Polymer Network for Drug-Releasing Medical Devices. J. Biomed. Mater. Res. - Part B Appl. Biomater. 2016, 104 (2), 402-410. https://doi.org/10.1002/jbm.b.33371.

(55) Steffensen, S. L.; Vestergaard, M. H.; Groenning, M.; Alm, M.; Franzyk, H.; Nielsen, H. M. Sustained Prevention of Biofilm Formation on a Novel Silicone Matrix Suitable for Medical Devices. Eur. J. Pharm. Biopharm. 2015, 94, 305-311. https://doi.org/10.1016/j.ejpb.2015.05.014.

(56) Xu, W. Z.; Li, X.; Charpentier, P. A. In Situ ATR-FT-IR Study of the Thermal Decomposition of Diethyl Peroxydicarbonate in Supercritical Carbon Dioxide. 2006. https://doi.org/10.1016/j.polymer.2006.12.018.

(57) and, F. M. R.; Giordani, S. Signal Processing at the Molecular Level. 2001. https://doi.org/10.1021/JA005699N.

(58) Wu, T.; Zou, G.; Hu, J.; Liu, S. Fabrication of Photoswitchable and Thermotunable Multicolor Fluorescent Hybrid Silica Nanoparticles Coated with Dye-Labeled Poly(NIsopropylacrylamide) Brushes. Chem. Mater. 2009, 21 (16), 3788-3798. https://doi.org/10.1021/cm901072g.

(59) Raymo, F. M.; Giordani, S. Signal Processing at the Molecular Level. J. Am. Chem. Soc. 2001, 123 (19), 4651-4652. https://doi.org/10.1021/ja005699n.

(60) Enhanced dopaminergic differentiation of human neural stem cells by synergistic effect of Bcl-xL and reduced oxygen tension - Krabbe - 2009 - Journal of Neurochemistry - Wiley Online Library https://onlinelibrary-wileycom.proxy.findit.dtu.dk/doi/epdf/10.1111/j.14714159.2009.06281.x (accessed Dec 18, 2019).

(61) Courtois, E. T.; Castillo, C. G.; Seiz, E. G.; Ramos, M.; Bueno, C.; Liste, I.; Martínez-Serrano, A. In Vitro and in Vivo Enhanced Generation of Human A9 Dopamine Neurons from Neural Stem Cells by Bcl-XL. J. Biol. Chem. 2010, 285 (13), 9881-9897. https://doi.org/10.1074/jbc.M109.054312.

(62) Shaw, D. J. Introduction to Colloid and Surface Chemistry; Elsevier, 2013. https://doi.org/10.1016/C2009-0-24070-0.

(63) Israelachvili, J. N. Intermolecular and Surface Forces; Acad. Press, 1992.

(64) Dupré, A.; Dupré, P. Théorie Mécanique de La Chaleur -; Gauthier-Villars: Paris, 1869.

(65) Hansen, C. M. Hansen Solubility Parameters; CRC Press, 2007. https://doi.org/10.1201/9781420006834. 
(66) Hansen, C.; Skaarup, K. Aspects of the Threedimensional Solubility Parameter. Dansk Kemi 1967, 48, 81-84.

(67) Beerbower, A. Surface Free Energy: A New Relationship to Bulk Energies. J. Colloid Interface Sci. 1971, 35 (1), 126-132. https://doi.org/10.1016/0021-9797(71)90192-5.

(68) Lide, D. R. CRC Handbook of Chemistry and Physics, 87th Edition; Taylor \& Francis, 2006.

(69) Barton, A. F. M. CRC Handbook of Solubility Parameters and Other Cohesion Parameters, Second Edition; Routledge, 2017. https://doi.org/10.1201/9781315140575.

(70) Van Krevelen, D. .; Te Nijenhuis, K. Properties of Polymers: Their Correlation with Chemical Structure; Elsevier Science, 2009.

(71) Determination of Interaction Parameters of Block Copolymers Containing Aromatic Polyesters from Solubility Parameters Obtained from Solution Viscosities. https://doi.org/10.1007/s00396-002-0728-6.

(72) Xue, R.; Zhang, X.; Tian, J. Synthesis of Water-Soluble Spiropyran-Modified Poly(Acrylic Acid) Micelles and Their Optical Behaviors. J. Photopolym. Sci. Technol. 2018, 31 (6), 739-746. https://doi.org/10.2494/photopolymer.31.739.

(73) Wu, T.; Zou, G.; Hu, J.; Liu, S. Fabrication of Photoswitchable and Thermotunable Multicolor Fluorescent Hybrid Silica Nanoparticles Coated with Dye-Labeled Poly( $\mathrm{N}$ Isopropylacrylamide) Brushes. Chem. Mater. 2009, 21 (16), 3788-3798. https://doi.org/10.1021/cm901072g.

(74) Tian, W.; Tian, J. An Insight into the Solvent Effect on Photo-, Solvato-Chromism of Spiropyran through the Perspective of Intermolecular Interactions. Dye. Pigment. 2014, 105, 66-74. https://doi.org/10.1016/j.dyepig.2014.01.020.

(75) Abdollahi, A.; Sahandi-Zangabad, K.; Roghani-Mamaqani, H. Light-Induced Aggregation and Disaggregation of StimuliResponsive Latex Particles Depending on Spiropyran Concentration: Kinetics of Photochromism and Investigation of Reversible https://doi.org/10.1021/acs.langmuir.8b02296.

(76) Abdollahi, A.; Alinejad, Z.; Mahdavian, A. R. Facile and Fast Photosensing of Polarity by Stimuli-Responsive Materials Based on Spiropyran for Reusable Sensors: A PhysicoChemical Study on the Interactions. J. Mater. Chem. C 2017,
6588 (5), 6588-6600. https://doi.org/10.1039/c7tc02232h.

Ritger, P. L.; Peppas, N. A. A Simple Equation for Description of Solute Release I. Fickian and Non-Fickian Release from Non-Swellable Devices in the Form of Slabs, Spheres, Cylinders or Discs. J. Control. Release 1987, 5 (1), 23-36. https://doi.org/10.1016/0168-3659(87)90034-4.

(78) Breitkreutz, J. Prediction of Intestinal Drug Absorption Properties by Three- Dimensional Solubility Parameters. Pharm. Res. 1998, $15 \quad$ (9), 1370-1375. https://doi.org/10.1023/A:1011941319327.

(79) Abbott, S. Solubility Science: Principles and Practice; TCNF Ltd: Ipswitch, 2017.

(80) Cunico, L. P.; Acosta, M. C.; Turner, C. Experimental Measurements and Modeling of Curcumin Solubility in CO2Expanded Ethanol. J. Supercrit. Fluids 2017, 130, 381-388. https://doi.org/10.1016/j.supflu.2017.06.018.

(81) Kajtez, J.; Buchmann, S.; Vasudevan, S.; Birtele, M.; Rocchetti, S.; Pless, C. J.; Heiskanen, A.; Barker, R. A. Martínez-Serrano, A.; Parmar, M.; Lind, J. U.; Emnéus, J. 3DPrinted Soft Lithography for Complex Compartmentalized Microfluidic Neural Devices. Adv. Sci. 2020, 7 (16), 2001150. https://doi.org/10.1002/advs.202001150.

(82) Courtois, E. T.; Castillo, C. G.; Seiz, E. G.; Ramos, M.; Bueno, C.; Liste, I.; Martínez-Serrano, A. In Vitro and in Vivo Enhanced Generation of Human A9 Dopamine Neurons from Neural Stem Cells by Bcl-X L. J. Biol. Chem. 2010, 285 (13), 9881-9897. https://doi.org/10.1074/jbc.M109.054312.

(83) Villa, A.; Snyder, E. Y.; Vescovi, A.; Martínez-Serrano, A. Establishment and Properties of a Growth Factor-Dependent, Perpetual Neural Stem Cell Line from the Human CNS. Exp. Neurol. $\quad 2000, \quad 161 \quad$ (1), 67-84 https://doi.org/10.1006/exnr.1999.7237.

\section{Insert Table of Contents artwork here}

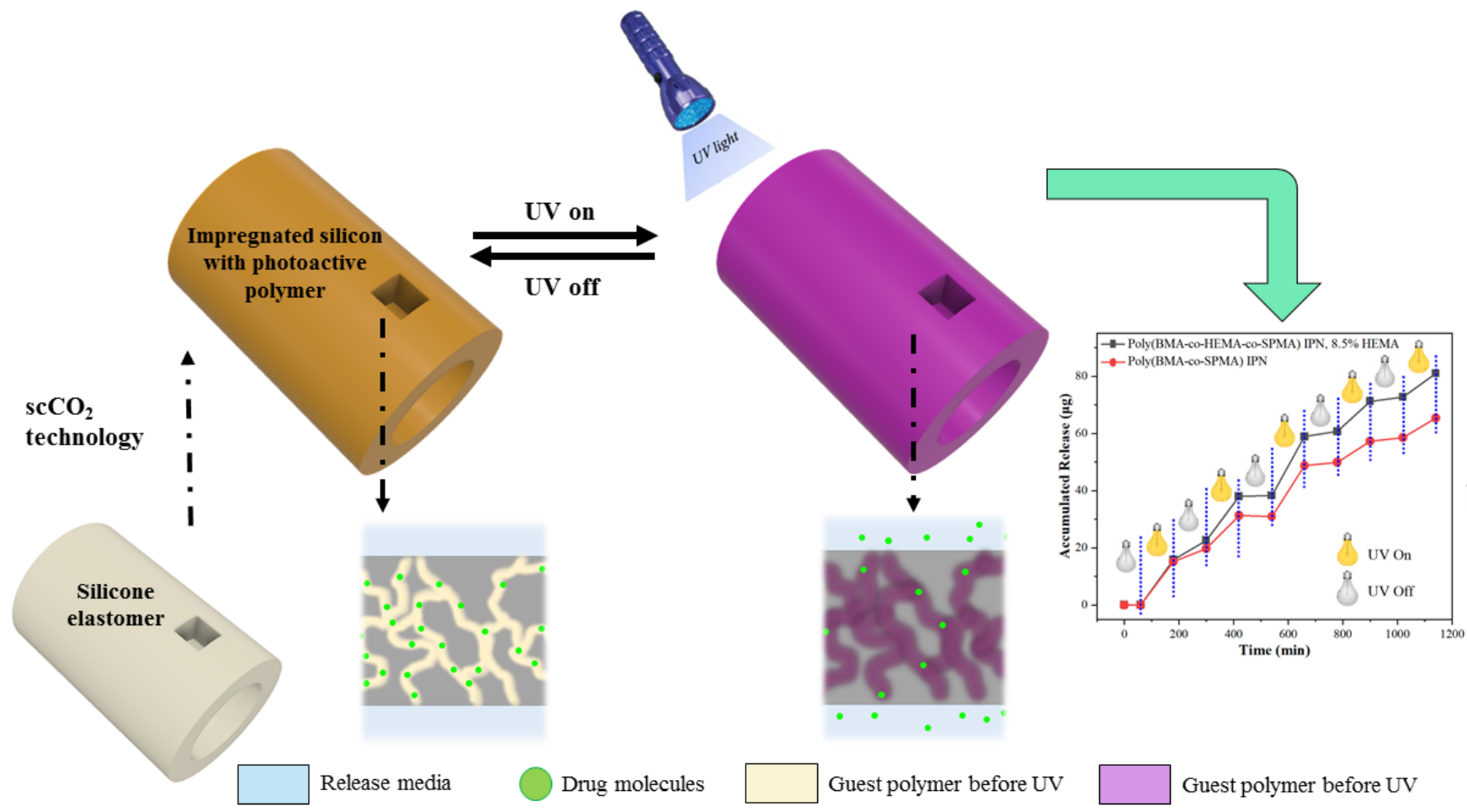

